\title{
Circuitos Disjuntos em Grafos
}

\author{
José Augusto Ramos Soares
}

Dissertação apresentada

ao

Instituto de Matemática e Estatistica

da

Universidade de São Paulo

para obtenção do grau de

Mestre em Matemática Aplicada

Área de concentração: Ciencia da Computação

Orientador: Paulo Feofiloff

- São Paulo, novembro de 1987 - 


\section{Fe șumo}

Este trabalho é motivado pelo seguinte problema: construir um algoritmo eficiente que ao receber um grafo $G$ e um nomero natural $k$ devolva uma coleção de $\mathrm{k}$ circuitos dois a dois disjuntos nos vértices ou alguma evidencia de que não existe uma tal coleçao.

o problema de decidir se um grafo tem $k$ circuitos disjuntos é NP-Campleto, a exemplo do que ocorre com os problemas análogos para circuitos orientados.

Apresentamos algumas condições suficientes e outras necessárias para que um grafo tenha $\mathrm{k}$ circuitos disjuntos. Demonstramos em seguida que para $k \geq 4$, todo grafo simples com numero de vértices $24 \mathrm{k}$ e grau minimo $22 \mathrm{k}-1$ tem $k$ circuitos disjuntos nos vértices, a menos que tenha uma 2-transversal de tamanho $<\mathrm{k}$. (Esta é uma extensão do teorema de Corradi e Hajnal [CH63] que resolve o problema quando o grau minimo do grafo é $2 \mathrm{kK}$.) A demonstração do teorema incuz um algoritmo, cujo tempo de execucão é $O\left(n^{3} \cdot m\right)$, onde $n$ é o número de vértices e $m$ o numero de arestas do grafo.

\section{Abstract}

This thesis is motivated by the problem of devising an efficient algorithm which, given a graph $G$ and a mumber $K$, would produce $k$ pairwise vertex-disjoint circuits in $G$ or some evidence that such a collection does not exist.

The problem of deciding if a graph has $\mathrm{k}$ disjoint circuits is IP-Complete, just as the analogous problems for directed graphs.

We discuss some sufficient conditions, as well as same necessary conditions, for the existence of $\mathrm{k}$ disjoint circuits in a graph. We then show that for $k \geq 4$ every simple graph with $\geq 4 \mathrm{k}$ vertices and minimum degree $22 k-1$ has $k$ vertex-disjoint circuits or a 2 -transversal of size $<\mathrm{k}$. (This is an extension of a theorem by Cormadi and Hajnal [CH63] that considered graphs with minimum degree $z 2 \mathrm{~K}$. ) The proof of our theorem induces an algorithm whise running time is $\mathrm{O}\left(\mathrm{n}^{3} \cdot \mathrm{m}\right)$, where $\mathrm{n}$ is the number of vertices nd $\mathrm{m}$ the number of edges of the graph. 


\section{inaice}

Capitulo 1 - o problema ................ 1

Duas tentativas inocentes de soluça do PCDV ......... 3

Algumas condiçరెes suficientes para a existência de $\mathrm{k}$ circuitos 4 Condições necessárias para a existencia de $\mathrm{k}$ circuitos:

o conceito de transversal .............. 7

Complexidade computacional ................... 9

Problemas correlatos ................ 10

Capitulo 2 - o teorema de Corrádi e Hajnal ......... 12

o teorema ....................... . . . 13

Complexidade computacional ............. 22

Capitulo 3 - Uma extensão do teorema de Corradi e Hajnal . . . 25

o teorema . . . . . . . . . . . . . . . . . 26

Complexidade computacional .............. 31

Apêndice . . . . . . . . . . . . . . . . . 33

Bibliografia . . . . . . . . . . . . . . . . 43

Este trabalho foi parcialmente suportado pela CAPES. 


\section{O problema}

A motivação central desta dissertação é o seguinte:

Problema dos Circuitos Disjuntos nos Vertices (PCDV): Dado um múmero inteiro $k$ e um grafo $G$, encontrar $k$ circuitos disjuntos nos vertices em $G$ ou alguma evidencia de que não existem os $k$ circuitos.

Mais especificamente, o que se deseja é um algoritmo eficiente que resolva o PCDV para todo $k$ e todo $G$.

Exemplo: Será que no grafo abaixo existem três circuitos disjuntos nos vértices? Uma verificaça cuidadosa nos mostra que a resposta é negativa.

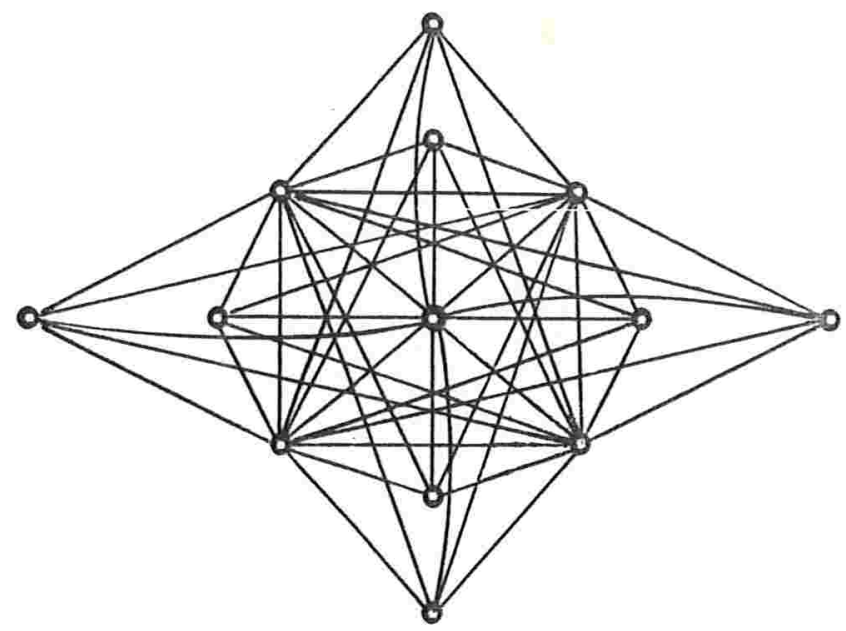

Vamos descrever aqui os conceitos usados no enunciado do PCDV . Um grafo consiste de um conjunto finito de vértices e outro conjunto de arestas, cada aresta sendo associada a um par não ordenado de vértices. O conjunto de vértices e arestas de um grafo G serão denotados por VG e AG, respectivamente.

Um circui o é um passeio que não tem vértices repetidos, exceto - primeiro e o último, que coincidem. Um passeio em um grafo G é uma seqüencia alternada de vértices e arestas, $\left(v_{0}, a_{1}, v_{1}, \ldots, a_{n}\right.$, $v_{n}$ ), tal que $a_{i}=v_{i-1} v_{i}$ para todo i entre 1 e $n$. Se $P$ é um passeio, VP e AP denotarão, respectivamente, o conjunto de vérti- 
ces e arestas de $P$. O comprimento de um circuito $C$ é a cardinalidade de VC. A cintura de um grafo G, $\gamma \mathrm{G}$, é o comprimento do menor circuito de G. Se G não tem circuitos, então $\gamma \mathrm{G}=+\infty$. Dois circuitos C e D são disjuntos nos vértices se VC $\cap$ VD $=\varnothing$. Diremos que $k$ circuitos são disjuntos se forem dois a dois disjuntos nos vertices.

O grau de um vértice $v$ em um grafo $G, g_{G}(v)$, é o número de arestas de $G$ incidentes em $v$. O grau minimo de um grafo $G$, $\delta G$, é o número $\min \left\{g_{G}(v): v \in V G\right\}$.

Podemos adiantar que a solução geral do PCDV é apenas uma meta desejável que, como veremos, dificilmente será alcançada. Este trabalho resolve 0 PCDV quando $\mathrm{k} \geq 4,|\mathrm{VG}| \geq 4 \mathrm{~K}, \gamma \mathrm{G} \geq 3 \mathrm{e}$ $\delta G 2$ 2K - 1. Esse é o resultado principal desta dissertacão, a ser apresentado no capitulo 3.

Uma variante do PCDV é a seguinte: dado um grafo $G$, determinar uma coleção máxima de circuitos disjuntos de G. Essa formulação não altera em muito a dificuldade do problema. Se tivermos um algoritmo que resolva eficientemente o PCDV, basta aplicar o algoritmo aos casos $\mathrm{k}=1, \mathrm{k}=2$, etc. Em no máximo |VG| + 1 passos obteriamos uma resposta negativa. A última resposta positiva corresponde então a uma coleção máxima de circuitos disjuntos. Reciprocamente, se soubermos encontrar uma coleção máxima de circuitos disjuntos, ficaremos sabendo qual a resposta do PCDV para qualquer valor de $\mathrm{k}$.

- PCDV tem solucão fácil no caso $\mathrm{k}=1$ : o problema se resume em verificar se um grafo tem ou não circuitos. Para $k=2$ o PCDV já é um pouco mais complicado, tendo sido resolvido em 1965 por Lovász [L065]. Para apresentar esse resultado é necessário definir o que é uma roda. Uma roda é um grafo $R$ que tem um vértice $v$ tal que $R-\{v\}$ consiste de um circuito e cada vértice de $R-\{v\}$ é adjacente a $\mathrm{v}$. Numa versão simplificada o resultado é o seguinte:

Todo grafo $G$ com $\gamma G \geq 3$ e $\delta G \geq 3$ tem dois circuitos disjuntos nos vertices, exceto se $G$ é uma roda ou é subgrafo de um dos grafos abaixo.
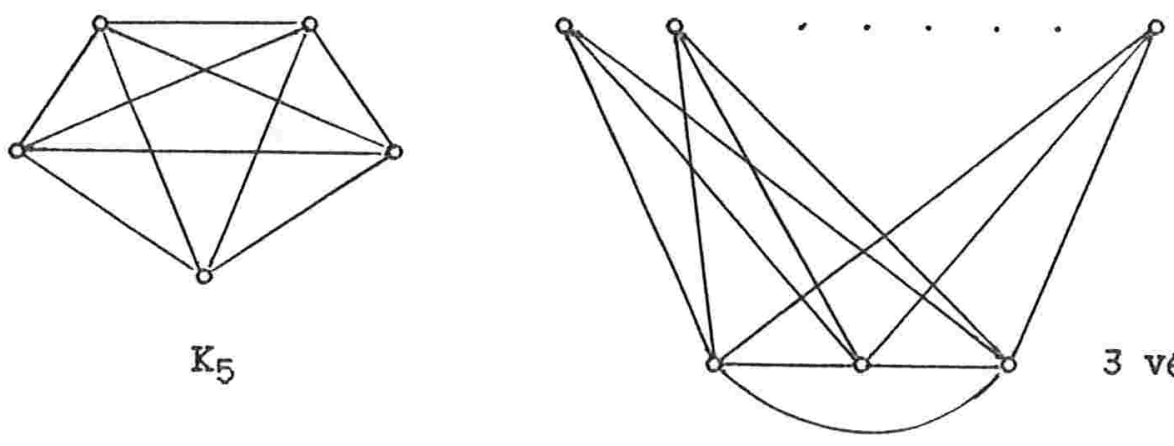

$|V G|-3$ vertices

Para o caso $\mathrm{k}=3$ ainda não se conhece algoritmo eficiente que resolva o problema e tampouco se conhece uma caracterização dos grafos que não têm 3 circuitos disjuntos. 
de solucáo do PCDV

Para resolver o PCDV sobre um dado grafo G, poderiamos tentar construir uma coleção de circuitos aleatoriamente, escolhendo um circuito, depois outro disjunto do primeiro, etc. Este algoritmo guloso pode, entretanto, produzir uma coleça arbitrariamente menor do que a máxima. Por exemplo, se o primeiro circuito escolhido no grafo abaixo for aquele definido pela seqüencia $(1,2, \ldots, 7,8)$, seremos forçados a escolher em seguida o circuito definido por $(9,10, \ldots, 24)$. Não há outro circuito disjunto destes dois. No entanto, o grafo tem 8 circuitos disjuntọs.

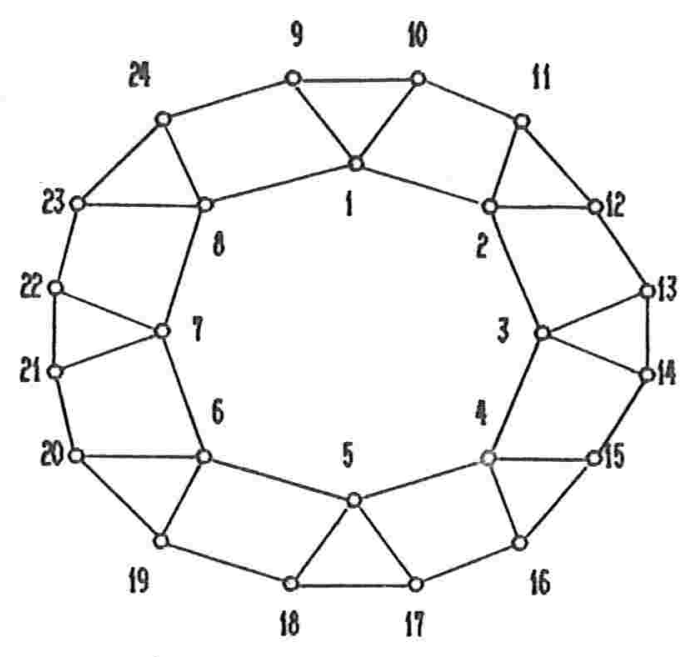

Numa segunda tentativa de solução do PCDV pode parecer interessante escolher o menor circuito disponivel a cada passo. O exemplo abaixo mostra que também esta estratégia não resolve o problema. 0 primeiro circuito a ser escolhido pode ser $(1,2,3)$; o segundo $(4,7$, 10). Obtemos dois circuitos. No entanto existem três circuitos disjuntos.

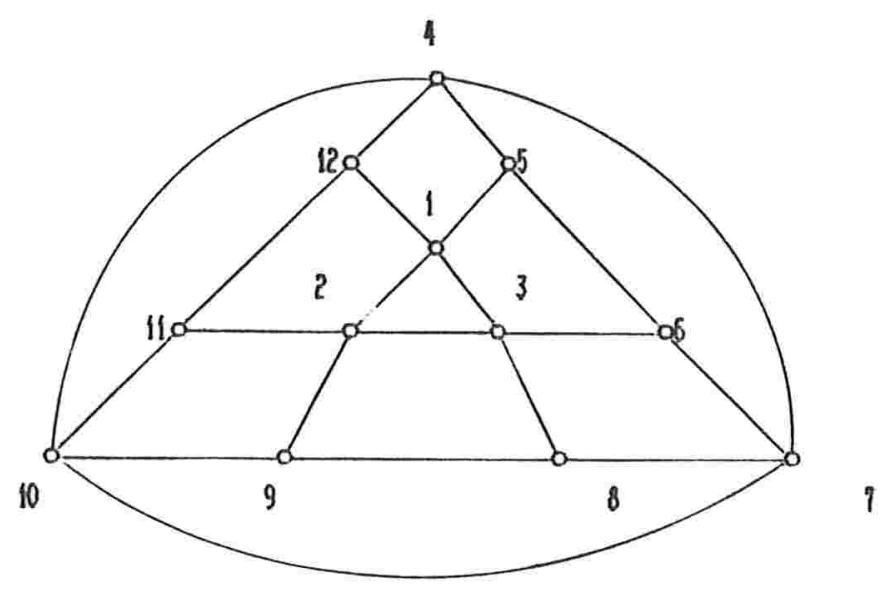


Embora não resolvendo o PCDV, o método exposto acima pode ser atil para deduzir condiçoses suficientes para um grafo ter $\mathrm{k}$ circuitos disjuntos: é o que veremos na próxima secão, em parte do capitulo 2 e na proposição A.1 do apendice.

\section{Algumas condigoes suficientes}

\section{para a existencia de k circuitos}

Vamos examinar algumas condições suficientes para a existência de $k$ circuitos disjuntos em um grafo. As proposiçóes abaixo, inspiradas no trabalho de Erdös e Pósa [EP62], dão uma seqüência de condições sobre a cintura e o numero de arestas do grafo; à medida que a restrição sobre a cintura é relaxada, as condicões sobre o número de arestas se tornam mais restritivas. No inicio do capitulo 2 veremos algumas condicões análogas sobre o par de parâmetros $\gamma, \delta$. A prova das validades das condições é interessante pois antecipa algumas das construções e raciocinios a serem usados nos capitulos 2 e 3 .

Precisaremos de algumas convenções de notação. Dado um grafo G e subconjuntos $\mathrm{X}$ e $\mathrm{Y}$ de $\mathrm{VG}, \mathrm{A}(\mathrm{X}, \mathrm{Y})$ é o conjunto das arestas de $G$ com uma ponta em $X$ e outra em $Y$. Dados $G$ e $X, G-X$ é o subgrafo gerado pelos vértices de VG - X .

Proposicão 1.1: Para qualquer inteiro $k \geq 1$, todo grafo $G$ tal que $\gamma G \geq 5,|V G| \geq 1$ e $|A G| \geq k \cdot|V G|$ tem $k$ circuitos disjuntos.

Prova (por indução em k): Para $\mathrm{k}=1$ a proposição é facilmente verificável: como se sabe, se $|A G|$ Z |VG|, então G tem circuitos. Vamos então verificar a proposição para um $\mathrm{k} \geq 2$.

Seja C um circuito de comprimento minimo de G. Seja $G^{\prime}=G-V C$. Como $|V C| \geq 5$, para todo $v$ em VG - VC vale $|A(V, V C)| \leq 1$, pois em caso contrário $V C U\{v\}$ inclui o conjunto de vértices de um circuito de comprimento menor do que o de $\mathrm{C}$. Temos então

$$
\begin{aligned}
|A G \cdot| & =|A G|-|A(V C, V G-V C)|-|A C| \\
& \geq|A G|-|V G-V C|-|V C|=|A G|-|V G| \\
& \geq k \cdot|V G|-|V G|=(k-1) \cdot|V G| \\
& \geq(k-1) \cdot|V G \cdot|
\end{aligned}
$$

e portanto, pela hipótese de indução, G' tem $\mathrm{k}-1$ circuitos disjuntos. Acrescentando-se $\mathrm{C}$ a esses circuitos obtem-se $\mathrm{k}$ circuitos disjuntos em G. 
Proposicão 1.2: Para qualquer inteiro $k \geq 1$, todo grafo $G$ tal que $\gamma G \geq 5,|V G| \geq 5 k$ e $|A G| \geq k \cdot|V G|-5 k \cdot(k-1) / 2$ tem $k$ circuitos disjuntos.

Prova: Seja $G_{1}=G$. Para $i=2,3, \ldots, r, r+1$, seja $C_{i-1}$ um circuito de comprimento minimo em $G_{i-1}$ e seja $G_{i} \circ$ grafo $G_{i-1}-C_{i-1}$. Repita a operação enquanto $G_{i}$ tiver circuitos. obtem-se dessa forma $r$ circuitos disjuntos em G. Queremos mostrar que $r \geq \mathrm{k}$.

Da mesma forma que na proposição anterior vale que $\left|\mathrm{AG}_{i+1}\right|$ $2\left|A G_{j}\right|-\left|V G_{j}\right|$ e portanto

$$
\left|A G_{j}\right| \leq\left|V_{i}\right|+\left|A G_{i+1}\right|, 1 \leq i \leq r \text {. }
$$

Como $G_{\Upsilon+1}$ não tem circuitos $\left|\mathrm{AG}_{\Upsilon+1}\right|<\left|\mathrm{VG}_{\Upsilon+1}\right|$. Então

$$
\begin{aligned}
\left|A G_{1}\right| & \leq\left|V G_{1}\right|+\left|A G_{2}\right| \leq\left|V G_{1}\right|+\left|V G_{2}\right|+\left|\mathrm{AG}_{3}\right| \leq \ldots \\
& \leq\left|V G_{1}\right|+\left|V G_{2}\right|+\left|V G_{3}\right|+\ldots+\left|V G_{1}\right|+\left|A G_{2+1}\right| \\
& <\left|V G_{1}\right|+\left|V G_{2}\right|+\left|V G_{3}\right|+\ldots+\left|V G_{r}\right|+\left|V G_{p+1}\right| .
\end{aligned}
$$

Como $\gamma G_{i} \geq 5$, temos $\left|V_{i+1}\right| \leq\left|V_{i}\right|-5$ para $1 \leq i \leq r$. Resulta que

$$
\begin{aligned}
\left|A G_{1}\right| & <\left|V G_{1}\right|+\left(\left|V G_{1}\right|-5\right)+\left(\left|V G_{1}\right|-10\right)+\ldots+\left(\left|V G_{1}\right|-5 r\right) \\
& \leq(r+1) \cdot\left|V G_{1}\right|-5 r \cdot(r+1) / 2 .
\end{aligned}
$$

Como $\left|A G_{1}\right| \geq \mathrm{k} \cdot|\mathrm{VG}|-5 \mathrm{k} \cdot(\mathrm{k}-1) / 2$, temos $\mathrm{k} \cdot\left|\mathrm{VG} \mathrm{G}_{1}\right|-5 \mathrm{k} \cdot(\mathrm{k}-1) / 2$ $<(r+1) \cdot\left|V G_{1}\right|-5 r \cdot(r+1) / 2$, donde

$$
5(\mathrm{k}+\mathrm{r}) \cdot(\mathrm{r}-\mathrm{k}+1) / 2<(\mathrm{r}-\mathrm{k}+1) \cdot\left|\mathrm{V} \mathrm{G}_{1}\right| .
$$

Concluimos que $r-k+1 \neq 0$. Se $r-k+1<0$, temos

$$
5(\mathrm{~K}+\mathrm{r}) / 2>\left|V G_{1}\right|=|V G| \geq 5 \mathrm{~K} \text {, }
$$

e portanto $r>k$, o que é inconsistente pois se $r>k$ então $r-k+1>0$. Portanto temos $r-k+1>0$, ou seja, $r \geq k$, como queriamos demonstrar.

Proposicão 1.3 [EP62]: Para qualquer inteiro $k \geq 1$, todo grafo $G$ t.a] que $\gamma G \geq 4,|V G| \geq 4 k$ e $|A G| \geq(2 K-1) \cdot|V G|-4 k \cdot(K-1)$ tem $K$ circuitos disjuntos.

Prova (por induç̃o em $\mathrm{k}$ ): Para $\mathrm{k}=1$ a proposiça é facilmente verificável. vamos então verificar a proposição para um $\mathrm{k} \geq 2$.

Suponna que $\gamma \mathrm{G}>4$. Como $|\mathrm{VG}| \geq 4 \mathrm{k}$, temos

$$
|A G| \geq(2 k-1) \cdot|V G|-4 k \cdot(k-1) \geq k \cdot|V G| \text {, }
$$

e a proposição 1.1 garante a existência de $k$ circuitos disjuntos em G . 
Supondo agora que $\gamma \mathrm{G}=4$, seja $\mathrm{C}$ um circuito de $\mathrm{G}$, de comprimento 4. Seja $G^{\prime}=G$ - VC. Note que como C é minimo e $|V C|=4$, para todo $v$ em $V G-V C$ vale $|A(v, V C)| \leq 2$, pois em caso contrário VC $U$ \{v\} inclui o conjunto de vértices de um circuito de comprimento menor do que o de C. Como $|A G '|=|A G|$ - $|\mathrm{A}(\mathrm{VC}, \mathrm{VG}-\mathrm{VC})|-|\mathrm{AC}|$, temos

$$
\begin{aligned}
|A G \cdot| & \geq|A G|-2|V G-V C|-|V C|=|A G|-2|V G|+4 \\
& \geq(2 k-1) \cdot|V G|-4 k \cdot(k-1)-2|V G|+4 \\
& \geq(2(k-1)-1) \cdot(\mid V G \cdot 1+4)-4 k \cdot(k-1)+4 \\
& \geq(2(k-1)-1) \cdot|V G \cdot|-4(k-1) \cdot(k-2)
\end{aligned}
$$

e portanto, pela hipotese de indução, G' tem $\mathrm{k}-1$ circuitos disjuntos. Acrescentando-se $\mathrm{C}$ a esses circuitos obtem-se $\mathrm{k}$ circuitos disjuntos em G .

Proposigão 1.4: Para qualquer inteiro $k \geq 1$, todo grafo $G$ tal que $\gamma G \geq 3,|V G| \geq 4 k$ e $|A G| \geq(3 k-2) \cdot|V G|-9 k \cdot(k-1) / 2$ tem $k$ circuitos disjuntos.

Prova (por indução em k): Para $\mathrm{k}=1$ a proposição é facilmente verificável. Vamos então verificar a proposicão para um $k \geq 2$.

Suponha que $\gamma G>3$. Como $|V G| \geq 4 \mathrm{~K}$ temos

$$
\begin{aligned}
|A G| & \geq(3 k-2) \cdot|V G|-9 k \cdot(k-1) / 2 \\
& \geq(2 k-1) \cdot|V G|-4 k \cdot(k-1),
\end{aligned}
$$

e a proposição 1.3 garante a existência de $\mathrm{k}$ circuitos disjuntos em G .

Supondo agora que $\gamma \mathrm{G}=3$, seja $\mathrm{C}$ um circuito de $\mathrm{G}$ de comprimento 3 . Seja $G^{\prime}=G-V C$. Para todo $v$ em VG - VC vale $|A(V, V C)| \leq 3$, pois $|\mathrm{VC}|=3$. Temos então

$$
\begin{aligned}
\left|A G^{\prime}\right| & =|A G|-|A(V C, V G-V C)|-|A C| \\
& \geq|A G|-3|V G-V C|-|V C|=|A G|-3|V G|+6 \\
& \geq(3 k-2) \cdot|V G|-9 k \cdot(k-1) / 2-3|V G|+6 \\
& \geq(3(k-1)-2) \cdot(|V G \cdot|+3)-9 k \cdot(k-1) / 2+6 \\
& \geq(3(k-1)-2) \cdot \mid V G \cdot 1-9(k-1) \cdot(k-2) / 2
\end{aligned}
$$

e portanto, pela hipotese de indução G' tem $k-1$ circuitos disjuntos. Acrescentando-se $\mathrm{C}$ a esses circuitos obtem-se $\mathrm{k}$ circuitos disjuntos em G. 
Os resultados obtidos acima possivelmente não são os melhores possiveis, com exceção da proposição 1.3: um grafo bipartido completo com $2 \mathrm{k}-1$ vértices de um "lado" tem $(2 \mathrm{k}-1) \cdot|\mathrm{VG}|-4 \mathrm{k} \cdot(\mathrm{k}-1)-1$ arestas e não tem $k$ circuitos disjuntos.

Erdös e Pósa [EP62], numa versão análoga à proposição 1.4, provam que todo grafo $G$ com $\gamma \mathrm{G} \geq 3,|\mathrm{VG}| \geq 24 \mathrm{~K}$ e $|\mathrm{AG}| \geq(2 \mathrm{~K}-1) \cdot|\mathrm{VG}|$ $-2 \mathrm{k}^{2}+\mathrm{k}+1$ tem $\mathrm{k}$ circuitos disjuntos. No apendice (proposiça A.6) mostramos, usando o teorema do capitulo 2 e a caracterização de Lovász do caso $k=2$ do PCDV, que todo grafo com $\gamma \mathrm{G} \geq 3$, $|V G| \geq 3 K$ e $|A G| \geq(2 k-1) \cdot|V G|-3\left(k^{2}+k\right) / 2+4$ tem $k$ circuitos disjuntos.

Nas proposições 1.2 a 1.4 e no resultado de Erdös aparecem restrições inferiores ao número de vértices do grafo. Este é um preço que é necessário pagar para se relaxar outras restrições. A restrição básica é muito natural: todo grafo $G$ que tenha $k$ circuitos disjuntos precisa necessariamente ter $|V G| \geq k \cdot \gamma G$. Mas há restrições mais severas, como é o caso da que aparece na proposiça 1.4 e no resultado de Erdös. Restrições semelhantes no número de vértices vão aparecer no teorema do capitulo 3.

\title{
Condicoses necessarias para a
}

\author{
existerncia de re circuitos: \\ o conceito de transversal
}

\begin{abstract}
Queremos condições necessárias para a existência de $\mathrm{k}$ circuitos disjuntos em um grafo, buscando assim uma caracterização eficiente da solução negativa do PCDV . Em jargão de Teoria de Complexidade a pergunta é: O PCDV está em co-NP ?

Vamos fazer um paralelo com o conhecido problema do fluxo máximo (Menger, 1927). Dado um grafo $G$, subconjuntos $X$ e $Y$ de VG, e um natural $\mathrm{k}$, encontrar $\mathrm{k}$ passeios dois a dois disjuntos nos vértices, ligando $\mathrm{X}$ a $\mathrm{Y}$. A condição necessária (e também suficiente) para a existência dos $\mathrm{k}$ passeios é dada em termos de transversais de passeios, também conhecidas como cortes de vértices: o problema tem solução se e só se todo corte que separa $X$ de $Y$ tem pelo menos $\mathrm{K}$ vértices.
\end{abstract}

A definição natural de transversal no caso do PCDV é a seguinte: uma transversal de circuitos em um grafo $G$ é um conjunt, $T$ de vertices tal que $\mid \mathrm{T} \cap \mathrm{VCl} \geq 1$ para qualquer circuito $\mathrm{C}$ de $\mathrm{G}$. Segue-se que uma condição necessária para a existência de $\mathrm{k}$ circuitos disjuntos é que $|T| z \mathrm{k}$ para toda transversal $\mathrm{T}$. Neste ponto é conveniente introduzir a seguinte definição: para todo grafo G, $T G$ é a cardinalidade da menor transversal de circuitos de G. A condição necessária pode agora ser expressa assim: $T \mathrm{G} \geq \mathrm{k}$. 
Observe que uma transversal é computacionalmente bem caracterizada: é fácil construir um algoritmo polinomial que decide se um dado conjunto de verrtices de um grafo é uma transversal de circuitos. Portanto, se a condição necessária acima fosse também suficiente, o PCDV estaria em co- $\mathbf{R}$.

Infelizmente a condição $T G \geq k$ não é suficiente, como pode ser constatado pelo seguinte exemplo, onde o grafo não tem 2 circuitos disjuntos mas $T G=2$ :

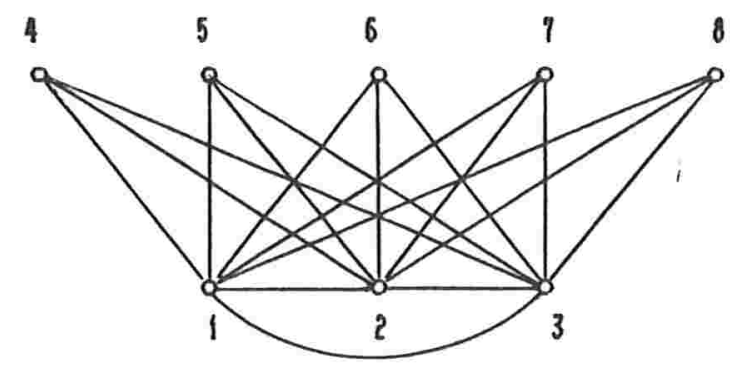

Erdös e Posa [EP65] mostram, entretanto, que

existe um numero positivo $c$ tal que todo grafo $G$ com $\tau G 2 c \cdot k \cdot l o g(k)$ tem $k$ circuitos disjuntos.

Erdös e Posa mostram mais: existe um número positivo d tal que para todo $k$ existe um grafo $G$ sem $k$ circuitos disjuntos tal que $\tau \mathrm{G} \geq \mathrm{d} \cdot \mathrm{k} \cdot \log (\mathrm{k})$.

Talvez a seguinte generalização do conceito de transversal ajude no tratamento do PCDV. Seja $t$ uma função definida nos vértices de um grafo $G$, com imagem em $\{0,1 / q, 2 / q, \ldots, q / q\}$, onde $q$ é um número inteiro positivo. Se para todo circuito $\mathrm{C}$ de $\mathrm{G}$ a soma dos valores de $t$ nos vertices de $C$ é maior ou igual a 1 , dizemos que $t$ é uma q-transversal de circuitos de G. O tamanho da q-transversal é a soma dos valores de $t$ para todos os vértices de G. Fixados $q$ e $G$, denotaremos por ${ }^{T} q^{G}$ o tamanho de qualquer q-transversal de tamanho minimo.

Todo grafo G com cintura $\gamma$ tem uma $\gamma$-transversal obvia de tamanho $|\mathrm{VG}| / \gamma$ : trata-se da transversal que atribui valor $1 / \gamma$ a cada vértice de $G$. Observe ainda que $\tau_{1} G=\tau G$ e $\tau_{q} G \leq \tau G$ para todo q 21 . Observamos, finalmente, que uma q-transversal é bem caracterizada computacionalmente: existe um algoritmo polinomial que decide se uma dada função $t$ é ou não uma q-transversal.

o conceito de q-transversal permite explicar, por exemplo, porque - grafo da ficura anterior não contém 2 circuitos disjuntos: a 2 -transversal fue associa aos vértices 1,2 e 3 o valor $1 / 2$ e aos demais vertices o valor 0 tem tamanho 1,5 ; como $1,5<2$, o grafo não pode ter dois circuitos disjuntos. Esta observação será generalizada no parágrafo seguinte. 
Se um grafo $G$ tem uma q-transversal de tamanho menor que $\mathbf{k}$, então $G$ não tem $k$ circuitos disjuntos. Isso porque todo circuito de G "gasta" pelo menos uma unidade de $T_{\mathrm{q}} \mathrm{G}$. Portanto, para todo $\mathrm{q}$, a condição $\mathrm{T}_{\mathrm{q}}^{\mathrm{G}} \geq \mathrm{K}$ é necessária para a existencia de $\mathrm{k}$ circuitos disjuntos $\mathrm{em} \mathrm{G}$.

Será que existe $q$ tal que a condição $\tau_{q} \geq k$ também é suficiente? A proposiça A.2 do apendice mostra uma familia de grafos (as rodas) onde isto não é verdade. A proposiç̃o A.3 mostra mais ainda: um grafo que não tem 3 circuitos disjuntos mas satisfaz $\tau_{q} G \geq 3$ para qualquer $q$.

Entretanto, para qualquer $\mathrm{k} \geq 4$, em todo grafo $\mathrm{G}$ com $\gamma \mathrm{G} \geq 3, \quad \delta \mathrm{G} \geq 2 \mathrm{~K}-1$ e $|\mathrm{VG}| \geq 4 \mathrm{~K}$, a condição $\mathrm{T}_{2} \mathrm{G} \geq \mathrm{K}$ é suficiente para a existencia de $\mathrm{k}$ circuitos. Este é o resultado central da dissertação, que veremos no capitulo 3 .

\section{Complexidade computacional}

E imediato constatar que um algoritmo que resolva o PCDV resolve tambem o problema abaixo.

Partigão em triângulos: Dado um grafo $G$ e um inteiro $q$ tais que $|V G|=3 q$, decidir se $G$ contem $q$ circuitos disjuntos nos vertices, sendo que cada circuito tem comprimento 3.

Conforme Garey e Johnson [GJ79, problema GT11], este problema é NP-Completo. Portanto, O PCDV (ou melhor, o problema de decisão correspondente ao $\mathbf{P C D V}$ ), que claramente está em $\mathbf{N P}$, também é $\mathbf{N P}-\mathrm{Com}-$ pleto.

Qual seria a complexidade do PCDV se considerássemos $\mathrm{k}$ como um dado fixo? Mais precisamente, teriamos o seguinte PCDVk : dado um grafo $G$, decidir se $G$ tem $k$ circuitos disjuntos nos vértices. Podemos afirmar que o PCDVk está em $\mathbf{P}$. E o que veremos a seguir.

Robertson e Seymour resolveram recentemente, numa série de artigos [RS86], o seguinte problema:

Dado um grafo $G$ e $k$ pares de seus vertices, $\left(s_{1}, t_{1}\right),\left(s_{2}, t_{2}\right)$, $\ldots,\left(s_{K}, t_{K}\right)$, determinar se existem passeios $P_{1}, P_{2}, \ldots, P_{K}$, dois a dois disjuntos nos vertices, cada $P_{i}$ ligando $s_{i} a$ $t_{i}$

Robertson e Seymour afirmam que resolvem esse problema através de um algoritmo cuja complexidadc é limitada por um polinómio em IVG| cujo grau não depende de $\mathrm{k}$ mas cujos coeficientes são funções exponenciais de $\mathrm{k}$. Considerando-se que o problema é NP-Completo [GJ79], o algoritmo de Robertson e Seymour é de certa forma o melhor possivel (a menos que $\mathbf{P}=\mathbf{N P}$ ). O valor prático do algoritmo é duvidoso: num recente artigo dedicado à análise dos resultados de Robertson e seymour, 
Johnson afirma [Jo87] que a constante que aparece como coeficiente do polinômio é pior que uma "torre de 2's", cuja altura (número de niveis de exponenciação) é pior do que exponencial em $\mathrm{k}$.

Podemos agora construir um algoritmo para o PCDVk cuja complexidade e limitada superiormente por um polinômio em IVG| cujo grau depende de $k$ e cujos coeficientes são funçoses exponenciais de $k$. Basta fazer o seguinte:

Para cada emparelhamento $\left\{s_{1} t_{1}, s_{2} t_{2}, \ldots, s_{K} t_{K}\right\}$ em $G$ remova de $\mathrm{G}$ as arestas do emparelhamento e submeta o grafo resultante, juntamente com os pares $\left(s_{1}, t_{1}\right),\left(s_{2}, t_{2}\right), \ldots,\left(s_{k}, t_{k}\right)$, ao algoritmo de Robertson e seymour. Se existirem os $k$ passeios, teremos também encontrado $\mathrm{k}$ circuitos disjuntos no grafo original. Se para cada um dos não mais que $|\mathrm{AG}|^{\mathrm{K}}$ emparelhamentos de $\mathrm{G}$ o algoritmo de Robertson e Seymour nos der uma resposta negativa, concluimos que o PCDVK não tem solução.

Na verdade Robertson afirma [Ro86] que tem um algoritmo para o PCDVK cuja complexidade é uma função de $|V G|^{3}$. Mas, devido ao inicialmente exposto, o algoritmo tem valor apenas teórico, pois é muito caro mesmo para valores moderados de $\mathrm{k}$. Portanto, a busca de um algoritmo eficiente, mesmo que exponencial em $\mathrm{k}$, é ainda um objetivo desejável não atingido.

\section{Prololemas correlatos}

Examinaremos a seguir alguns problemas semelhantes ao PCDV que têm recebido atenção na teoria dos grafos. Curiosamente não existem laços fortes que relacionem esses problemas com o nosso.

O primeiro problema é o PCDA (problema dos circuitos disjuntos nas arestas): dado um grafo $G$ e um inteiro $\mathrm{k}$, encontrar $\mathrm{k}$ circuitos dois a dois disjuntos nas arestas em G O O PCDA não é completamente independente do PCDV, pois circuitos disjuntos nos vértices são também disjuntos nas arestas. Ademais, para grafos de grau máximo 3 , o PCDA e o PCDV são equivalentes, pois dois circuitos disjuntos nas arestas são também, necessariamente, disjuntos nos vértices. Não sabemos qual é a complexidade computacional do PCDA. Redefinindo adequadamente $\circ$ conceito de transversal e o parâmetro $T$ para $\circ$ PCDA , vale repetir as perguntas que fizemos na seção "Condições necessárias...": será que $\tau \geq \mathrm{K}$ é condição suficiente para a existência de $\mathrm{k}$ circuitos disjuntos nas arestas? O grafo abaixo, que não tem dois circuitos disjuntos e para o qual $\tau=3$, mostra que a resposta à pergunta é "não". 


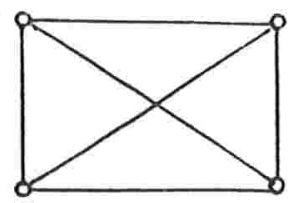

Um outro problema correlato é o PCODV (problema dos circuitos orientados disjuntos nos vértices): dado um grafo orientado $G$ e um inteiro $\mathrm{k}$ encontrar $\mathrm{k}$ circuitos orientados disjuntos nos vértices em G. Este problema é IP-Completo, conforme proposição A.4 do apêndice.

Como indica Thomassen [Th83], é possivel que exista um algoritmo para o PCODV cuja complexidade computacional é dada por um polinómio em $|V G|$ cujo grau é funcão de $\mathrm{k}$. Isto depende da verificacão da seguinte conjectura [BT81] formulada por Younger em 1973:

Existe uma funcão h tal que todo grafo orientado $G \mathrm{com}$ $\tau G \geq h(k)$ tem $k$ circuitos orientados disjuntos nos vertices.

E claro que neste enunciado $T G$ é o tamanho de uma transversal minima de circuitos orientados. Observe que a versão da conjectura para grafos não orientados é teorema: como já indicamos acima, Erdös e Pósa [EP65] mostraram que neste caso $h(\mathrm{k})=\mathrm{c} \cdot \mathrm{K} \cdot \log (\mathrm{K})$.

A conjectura de Younger já foi demonstrada para algumas familias de grafos. Assim, Szwarcfiter [Sz87] prova que para todo grafo G conexamente redutivel $T G \geq \mathrm{K}$ é condição necessária e suficiente para a existência de $\mathrm{k}$ circuitos orientados disjuntos nos vértices em $\mathrm{G}$ (h neste caso é a funcão identidade).

- PCODA - o problema de encontrar circuitos orientados disjuntos nas arestas - é equivalente ao PCODV ; qualquer algoritmo polinomial que resolva um dos problemas pode ser transformado num algoritmo polinomial que resolve o outro. Esta redução se encontra descrita na proposição A.5 do apendice.

A versão da conjectura de Younger para circuitos orientados disjuntos nas arestas em grafos orientados planares é teorema: a condição $T G \geq \mathrm{K}$ (com $T G$ adequadamente redefinido) é necessária e suficiente para a existência dos $k$ circuitos disjuntos nas arestas. Isto foi demonstrado por Lucchesi e Younger [LY78]. Com base nesta condição é possivel construir um algoritmo polinomial que resolve o PCODA para grafos orientados planares.

Outros resultados e conjecturas sobre circuitos orientados podem ser encontrados na resenha de Bermond e Thomassen [BT81]. 


\section{O teorema de}

\section{Corrádi e Hajnal}

Usaremos no restante deste trabalho a seguinte notaça e terminologia: Se $\mathbf{C}$ é uma coleção de circuitos, então VC é a união dos conjuntos de vértices dos circuitos de $\mathbf{C}$. AC é a união dos conjuntos de arestas dos circuitos de $\mathbb{C}$. Um caminho é um passeio sem vértices repetidos. Uma corda de um circuito $\mathrm{C}$ é uma aresta que liga dois vértices não consecutivos de $\mathrm{C}$. Um triângulo ( quadrado ) é um circuito que tem exatamente 3 ( 4 ) vértices. Um vértice $v$ é folha de um grafo $\mathrm{H}$ se $\mathrm{G}_{\mathrm{H}}(\mathrm{v})=1$.

Como introdução ao teorema de Corradi e Hajnal iremos apresentar algumas condições suficientes sobre $\gamma G$ e $\delta G$ para a existência de $k$ circuitos disjuntos.

Proposiç̃ 2.1: Para qualquer inteiro $k \geq 1$, todo grafo $G$ tal que $|V G| \geq 1, \gamma G \geq 5$ e $\delta G \geq k+1$ tem $K$ circuitos disjuntos.

Prova (por indução em k): Para $\mathrm{k}=1$ a proposição é facilmente verificável. Vamos então verificar a proposição para um $k \geq 2$.

Seja C um circuito de comprimento minimo de G. Seja $G^{\prime}=\mathrm{G}-\mathrm{VC}$. Como $\mathrm{C}$ não tem cordas (por ter comprimento minimo) e $\delta G \geq 3$, podemos garantir que $|V G '| \geq 1$. Como $|V C| \geq 5$, para todo $\mathrm{v}$ em $\mathrm{VG}$ - VC vale $|\mathrm{A}(\mathrm{V}, \mathrm{VC})| \leq 1$, pois em caso contrário VC $U\{v\}$ inclui o conjunto de vértices de um circuito de comprimento menor do que o de $\mathrm{C}$. Portanto $\delta G^{\prime} \geq(\mathrm{K}+1)-1$. Obviamente $\gamma \mathrm{G}^{\prime} \geq \gamma \mathrm{G}$ 25 , garantindo assim que podemos aplicar a hipótese de indução a G' para obter $\mathrm{k}-1$ circuitos disjuntos. Acrescentando-se $\mathrm{C}$ a esses circuitos obtem-se $\mathrm{k}$ circuitos disjuntos em $\mathrm{G}$.

Proposigão 2.2: Para qualquer inteiro $k \geq 1$, todo grafo $G$ tal que $|V G| \geq 1, \gamma G \geq 4$ e $\delta G \geq 2 k$ tem $k$ circuitos disjuntos.

Proposicão 2.3: Para qualquer inteiro $k \geq 1$, todo grafo $G$ tal que $|V G| \geq 1, Y G \geq 3$ e $\delta G \geq 3 k-1$ tem $k$ circuitos disjuntos. 
As provas dessas proposiços são semelhantes a prova da proposição 2.1. A diferença é que na proposição 2.2 temos que $|\mathrm{A}(\mathrm{v}, \mathrm{VC})| \leq 2$, e na $2.3|\mathrm{~A}(\mathrm{v}, \mathrm{VC})| \leq 3$, para todo $\mathrm{v}$ em VG - VC .

o caso $\gamma \geq 2$ (arestas multiplas) apresenta uma particularidade que não aparece no caso $\gamma \geq 3$ : mais do que duas arestas em paralelo podem aumentar arbitrariamente o grau de um vértice sem trazer nenhuma contrapartida em relação ao numero de circuitos disjuntos do grafo. Podemos, portanto, não considerar mais do que duas arestas em paralelo para cada par de vértices. Com essa restrição é fácil deduzir, de forma análoga às proposições acima, que todo grafo $G$ com $|V G| \geq 1$, $\gamma G \geq 2$ e $\delta G \geq 4 \mathrm{~K}-2$ tem $\mathrm{k}$ circuitos disjuntos.

Corrádi e Hajnal melhoraram [CH63] as condições da proposição 2.3 mostrando que todo grafo $G$ com $|V G| \geq 3 \mathrm{~K}, \gamma \mathrm{G} \geq 3$ e $\delta G \geq 2 \mathrm{~K}$ tem $\mathrm{k}$ circuitos disjuntos. Como sugerem as proposiçőes 2.1 a 2.3 a dificuldade do problema advem dos circuitos curtos, especialmente dos triângulos. Isso fica particularmente agravado quando o grafo tem somente 3k vertices. (Tanto assim que a prova de Corrádi e Hajnal tem duas partes, uma para o caso $|V G|>3 \mathrm{k}$ e outra para $|V G|=3 \mathrm{k}$.)

Neste capitulo é apresentada uma demonstração do teorema de Corrádi e Hajnal, que não difere no essencial da originalmente apresentada por eles. Como no capitulo 3 apresentaremos uma extensão deste teorema, pretendemos aqui atingir dois objetivos. Primeiro, apresentar os dois teoremas de modo uniforme. Segundo, introduzir as dificuldades que serão encontradas no próximo capitulo. Acreditamos também que esta demonstração é mais simples e legivel do que a feita por Corrádi e Hajnal.

Bollobás [Bo78] sugere uma demonstração alternativa deste teorema, que parece ser mais "limpa" do que a aqui apresentada. Porém não vemos como aplicar o mesmo método para obter o resultado do capitulo 3.

\section{O Teorema}

Teorema 2.1 [CH63]: Fara qualquer inteiro $K \geq 1$, todo grafo $G$ tal que $\gamma G \geq 3, \delta G \geq 2 \mathrm{~K}$ e ${ }^{{ }_{3} G} \geq \mathrm{K}$ tem $\mathrm{k}$ circuitos disjuntos.

A condição $\tau_{3} G \geq \mathrm{K}$ implica $|\mathrm{VG}| \geq 3 \mathrm{~K}$, pois em grafos com menos verrtices podemos definir uma 3-transversal atribuindo valor $1 / 3$ a cada um dos vértices do grafo. E a condição IVG| $23 \mathrm{~K}$ é claramente necessária, uma vez que $\gamma \mathrm{G} 2{ }^{7}$. No mais, o teorema pode ser reduzido ao seguinte lema: 
Lema 2.1: Seja $k$ um natural $\geq 1$. Para qualquer grafo $G$ tal que. $|V G| \geq 3 K, \quad \gamma G \geq 3$ e $\delta G \geq 2 K$ e para qualquer coleça $C$ de circuitos disjuntos tal que $|\boldsymbol{C}|<K$, existe uma colecão $\boldsymbol{C}^{\prime}$ de circuitos disjuntos que satisfaz uma das seguintes condicóes:
(a) $\left|\mathbb{C}^{\prime}\right|>|C|$;
(b) $\left|\mathbb{C}^{\prime}\right|=|\mathbb{C}|$ mas $\left|\mathrm{VH}^{\prime}\right|>|\mathrm{VH}|$
(c) $\left|C^{\prime}\right|=|C|$ e $\left|V^{\prime}\right|=|V H|$ mas $\left|A H^{\prime}\right|>|A H|$,
onde $H=G-V \mathbb{C}$ e $H^{\prime}=G-V C^{\prime}$.

o lema sugere o seguinte processo iterativo: começa-se com uma coleção de circuitos (possivelmente vazia); usa-se o lema repetidas vezes, cada vez começando com a coleção $\mathbf{C}^{\prime}$ fornecida pela chamada anterior. As alternativas (b) e (c) não podem se repetir indefinidamente. Após um numero finito de iterações, a condição (a) é satisfeita. Este processo prova o teorema a partir do lema.

Prova do Lema: A prova do lema terá um caráter algoritmico e portanto vamos nos referir às condições (a), (b) e (c) como "objetivos" do lema. Seja $H=G-V C$. Se $H$ tem um circuito, digamos C, então o objetivo (a) está satisfeito $\mathrm{com} \quad \mathbb{C}^{\prime}=\mathbb{C} U\{\mathrm{C}\}$ e portanto a prova terminou. Vamos pois supor no que se segue que $H$ é uma floresta.

A prova do lema se desdobra basicamente em 5 casos, dependendo do tamanho de $\mathrm{H}$ e do grau de seus vertices. Antes de considerar os 5 casos é conveniente descartar a possibilidade de se atingir imediatamente o objetivo (b): suponha que existe $\mathrm{C}$ em $\mathbb{C}$ e $\mathrm{v}$ em $\mathrm{VH}$ tais que VC $U\{\mathrm{v}\}$ contém um conjunto de vértices de um circuito $\mathrm{C}^{\prime}$ menor que $\mathbf{C}$. Neste caso, troque $\mathrm{C}$ por $\mathrm{C}^{\prime}$ em $\mathbb{C}$ atingindo o objetivo (b). O mesmo pode ser feito se C tem corda.

Vamos considerar no restante desta prova que a configuração considerada no parágrafo anterior não existe. Assim, para todo $\mathrm{C}$ em $\mathbb{C}$ e todo $\mathrm{h}$ em $\mathrm{VH}, \mathrm{C}$ não tem cordas e pelo menos uma das seguintes afirmações é verdadeira:

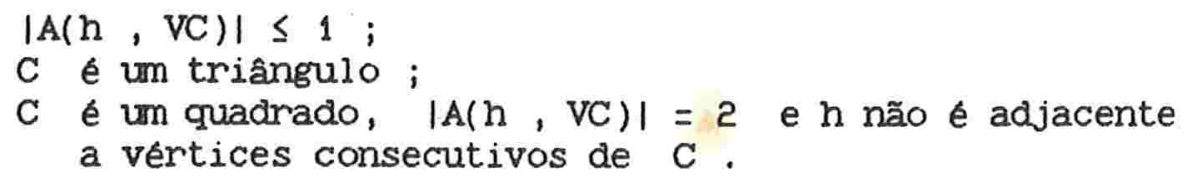

A prova se desdobra agora em 5 casos.

Caso 1: $|\mathrm{VH}|<3$.

Seja C um circuito de $\mathbb{C}$ tal que $|\mathrm{VC}|$ é máximo. Como

$$
|V \mathbb{C}|=|V G|-|V H| \geq 3 \mathrm{~K}-2>3(\mathrm{k}-1) \geq 3|\mathbb{C}|
$$

devemos ter $|\mathrm{VC}|>3$. Como $\mathrm{C}$ não t€ $\mathrm{n}$ cordas, vale $|\mathrm{A}(\mathrm{C}, \mathrm{VG}-\mathrm{VC})|$ $\geq \delta G-2 \geq 2 K-2$ para cada $c$ em VC, donde

$$
|\mathrm{A}(\mathrm{VC}, \mathrm{VG}-\mathrm{VC})| \geq|\mathrm{VC}| \cdot(2 \mathrm{k}-2) \text {. }
$$


Por outro lado, $|A(V C, h)| \leq 2$ para cada $h$ em $V H$ em virtude da hipotese (*). Segue-se que

$$
\begin{aligned}
|\mathrm{A}(\mathrm{VC}, \mathrm{VC}-\mathrm{VC})| & \geq|\mathrm{VC}| \cdot(2 \mathrm{k}-2)-2|\mathrm{VH}| \\
& \geq|\mathrm{VC}| \cdot(2 \mathrm{k}-2)-4 \\
& >2|\mathrm{VC}| \cdot(\mathrm{k}-2) \\
& \geq 2|\mathrm{VC}| \cdot|\mathrm{C}-\{\mathrm{C}\}| .
\end{aligned}
$$

Portanto existe $D$ em $\mathbf{C}-\{C\}$ tal que $|\mathrm{A}(\mathrm{VC}, \mathrm{VD})| \geq 2|\mathrm{VC}|+1$. Pela maximalidade de $C$, vale também $|A(V C, V D)| z$ 2|VD| +1 . Essas desigualdades garantem a existencia de $v$ em $v C e$ w em VD, tais que $|A(v, V D)| \geq 3$ e $|A(w, V C)| \geq 3$.

Iremos usar a seguinte notação: sé $F$ é um circuito $\left(v_{0}, a_{1}\right.$, $\left.v_{1}, \ldots, a_{n}, v_{n}\right)$, então $F\left[v_{i}, v_{j}\right]=\left\{v_{i}, v_{i+1}, \ldots, v_{j-1}, v_{j}\right\}$. Se trocamos o colchete inicial pelo parentese, devemos eliminar $v_{i}$ do conjunto, convencão análoga valendo para o colchete final.

Ajuste a notaç̃o de $D$ de modo que a origem de $D$ seja o primeiro vizinho de $v$ em $v D$ que precede $w$ (possivelmente a origem seja w ). Sejam $w_{0}, w_{1}, w_{2}, \ldots$ todos os vizinhos de $v$ em vD, na ordem em que aparecem em $D$. Portanto $w \in D\left[w_{0}, w_{1}\right)$. De forma análoga ajuste a notaça de $\mathrm{C}$, e sejam $\mathrm{v}_{0}, \mathrm{v}_{1}, \mathrm{v}_{2}, \ldots$ todos os vizinhos de $w$ em VC, na ordem em que aparecem em $C$, com $v \in C\left[v_{0}, v_{1}\right)$. Seja C' o circuito com vertices em $C\left[v_{1}, v_{2}\right] U\{w\}$ e $D^{\prime}$ o circuito com vértices em $D\left[w_{1}, w_{2}\right] \cup\{v\}$. Pela definiç̃o acima, $C^{\prime}$ e $D^{\prime}$ são disjuntos. $C^{\prime}$ não e maior que $C$, pois apesar de $w$ não pertencer a VC, pelo menos o vértice $v$ não está em $C^{\prime}$. O mesmo vale para $D^{\prime}$ em relação a $D$.
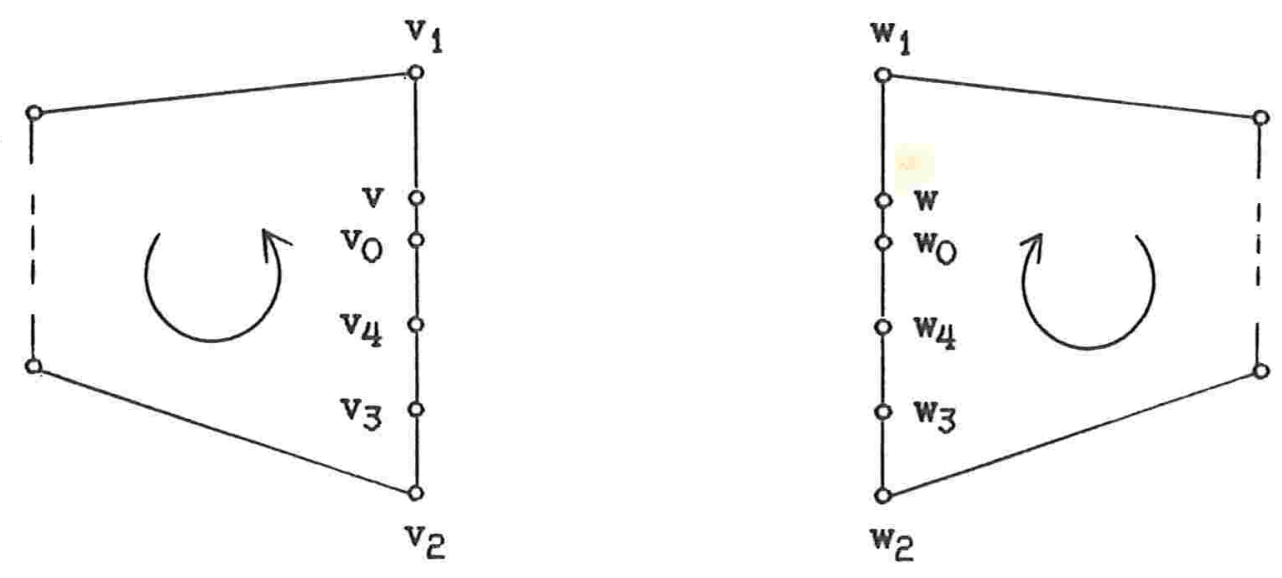

Se $|\mathrm{VC}|<|\mathrm{VC}|$ ou $\left|\mathrm{V} \mathrm{D}^{\prime}\right|<|\mathrm{VD}|$ o objetivo (b) esti atingido. Caso contrário é fácil ver que $\mathrm{v}=\mathrm{v}_{0}$ e $\mathrm{w}=\mathrm{w}_{\mathrm{O}}$, e mais ainda, todos os vértices de $C$, exceto $v$, estão em $C\left[v_{1}, v_{2}\right]$ e todos os vértices de $D$, exceto $w$, estão em $D\left[w_{1}, w_{2}\right]$. Com isso $v$ tem somente tres vizinhos em $D$, o mesmo ocorrendo com w em relasão a $C$. 

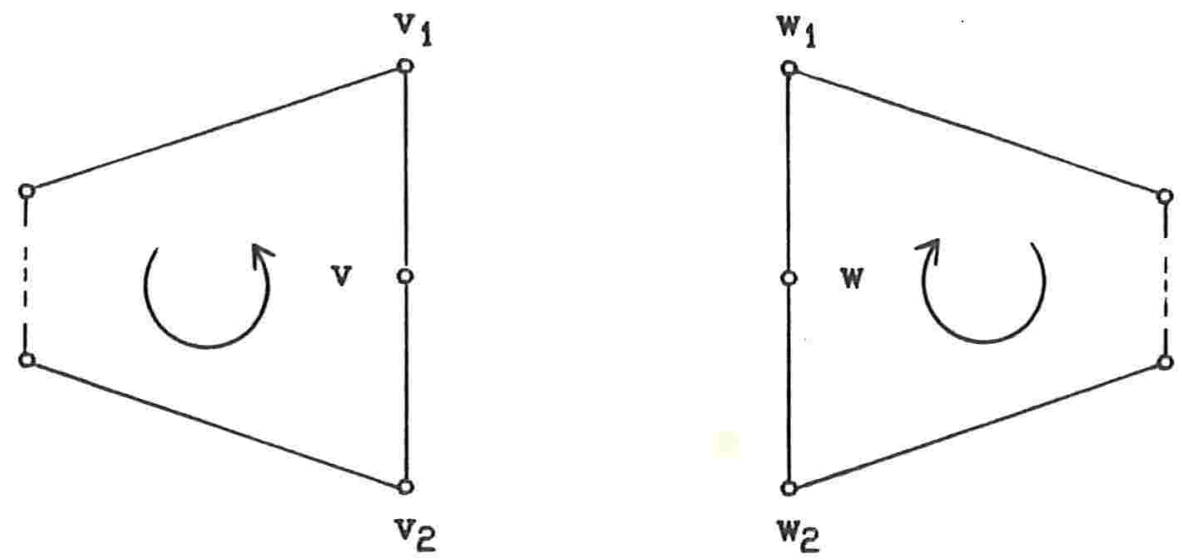

Além das arestas $v w, v w_{1}, v w_{2}, w v_{1} e w v_{2}, o$ conjunto $A(V C, v D)$ contem mais $2 \mid \mathrm{VCl}+1-5$ arestas, nenhuma delas com ponta em $v$. Como $2|\mathrm{VC}|-4=|\mathrm{VC}|+|\mathrm{VC}|-4>|\mathrm{VC}|-1$, existe um vértice $v_{3}$ de $C$ com dois ou mais vizinhos em $D$, sem contar as arestas $w v_{1} e w_{2}$. Sejam $w_{3} e w_{4}$ dois desses vizinhos, na ordem que aparecem em $\mathrm{D}$. Como $w$ so é adjacente a $v_{0}, v_{1} e v_{2}$, concluimos que $w_{3}$ e $w_{4}$ estão em $D\left[w_{1}, w_{2}\right]$. Seja $D^{\prime}$ o circuito com vértices em $\left\{v_{3}\right\} \cup D\left[w_{3}, w_{4}\right]$. $D^{\prime}$ não é maior do que $D$, pois pelo menos $w$ não está em $D\left[w_{4}, w_{5}\right]$.

Se $v_{3}=v_{1}$ então seja $C^{\prime} \circ$ triângulo com vértices em $\{w, v$, $\left.v_{2}\right\}$. Caso contrário seja $C^{\prime}$ o circuito com vértices em $\left\{w, v, v_{1}\right\}$. Esta definição de $C^{\prime}$ visa garantir que o mesmo seja disjunto de $D^{\prime}$. Temos $|\mathrm{VC}| \geq 4>3=\left|\mathrm{VC}^{\prime}\right|$. A coleção $(\mathbb{C}-\{C, D\}) \cup\left\{C^{\prime}, D^{\prime}\right\}$ satisfaz o objetivo (b) do lema.

Caso 2: $|\mathrm{VH}|=3$ e $\mathrm{g}_{\mathrm{H}}(\mathrm{h}) \geq 1$ para todo $\mathrm{h}$ em $\mathrm{H}$.

Observe que $H$ é um caminho cujo vértice interno, digamos $h_{2}$, tem grau 2 em $\mathrm{H}$ e os extremos grau 1. Logo

$$
\begin{aligned}
|A(V H, V C)| & \geq(\delta G-1)+(\delta G-2)+(\delta G-1) \\
& >6(K-1) \\
& \geq 6|\mathbb{C}| .
\end{aligned}
$$

Portanto existe $\mathrm{C}$ em $\mathbb{C}$ tal que $|\mathrm{A}(\mathrm{VH}, \mathrm{VC})|>6$ e $|\mathrm{VC}|=3$, de acordo com a hipotese (*). Se VH U VC for união disjunta dos vértices de dois circuitos, digamos $C^{\prime}$ e $C^{\prime \prime}$, então a coleção $(\mathbb{C}-\{C\}) \cup\left\{C^{\prime}, C^{\prime \prime}\right\}$ satisfaz o objetivo (a) do lema. Suponhamos no que segue que $\mathrm{VH} U$ VC não é união disjunta dos vertices de dois circuitor. Então a relação entre $\mathrm{H}$ e $\mathrm{C}$ é a seguinte: 
H

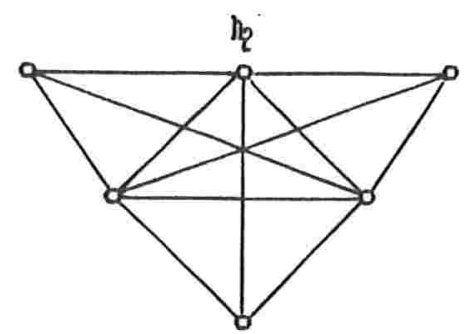

Observe que

$$
\begin{aligned}
\left|A\left(V H-\left\{h_{2}\right\}, V C-V C\right)\right| & \geq(\delta G-5+2)+(\delta G-5+2) \\
& >4(k-2) \geq 4|C-\{C\}| .
\end{aligned}
$$

Portanto existe $D$ em $\mathbf{C}-\{C\}$ tal que $\left|A\left(V H-\left\{h_{2}\right\}, V D\right)\right|>4 . E m$ virtude da hipótese (*), $|V D|=3$ donde $\mid A(h$, VD)| $\leq 3$ para cada $h$ em VH - $\left\{h_{2}\right\}$. Sejam $h_{1}$ e $h_{3}$ os dois elementos de $V_{H}-\left\{h_{2}\right\}$, definidos de tal modo que $\left|A\left(h_{1}, V D\right)\right|=3$ e $\left|A\left(h_{3}, V D\right)\right| \geq 2$. Sejam $d_{1}, d_{2}$ e $d_{3}$ os vértices de $D, c_{1}, c_{2}$ e $c_{3}$ os vértices de $C$ definidos de acordo com a figura abaixo:

D

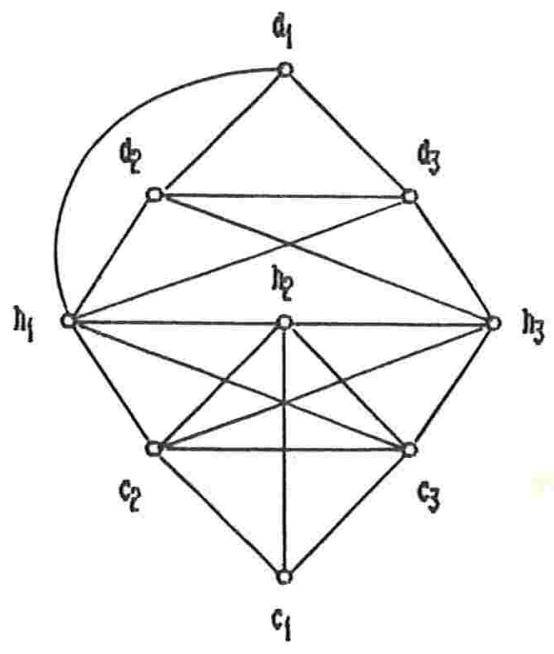

subcaso 2.1: $h_{2}$ é adjacente a $d_{1}, d_{2}$ ou $d_{3}$;

ou $c_{2}$ e adjacente $a d_{2}$.

Em cada um dos quatro casos VC U VD U VH é união disjunta dos vértices de 3 circuitos, digamos C', C", C'"', e portanto a coleção $(\mathbb{C}-\{C, D\}) \cup\left\{C^{\prime}, C^{\prime \prime}, C^{\prime \prime}\right\}$ satisfaz o objetivo (a) do lema. 

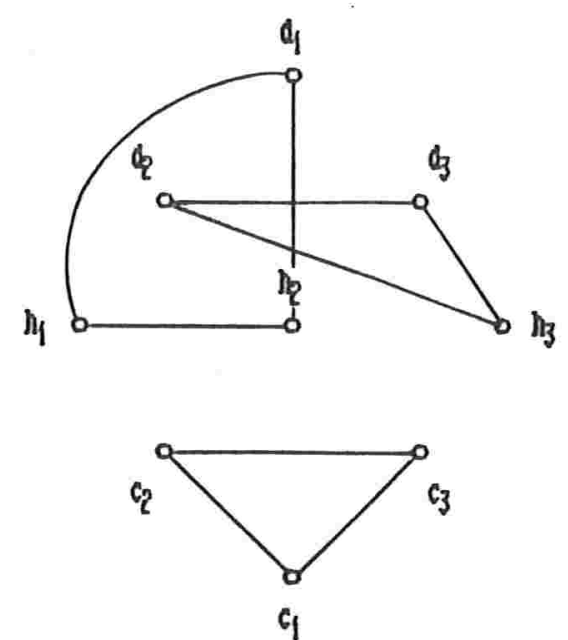

$h_{2}$ adjacente a $d_{1}$
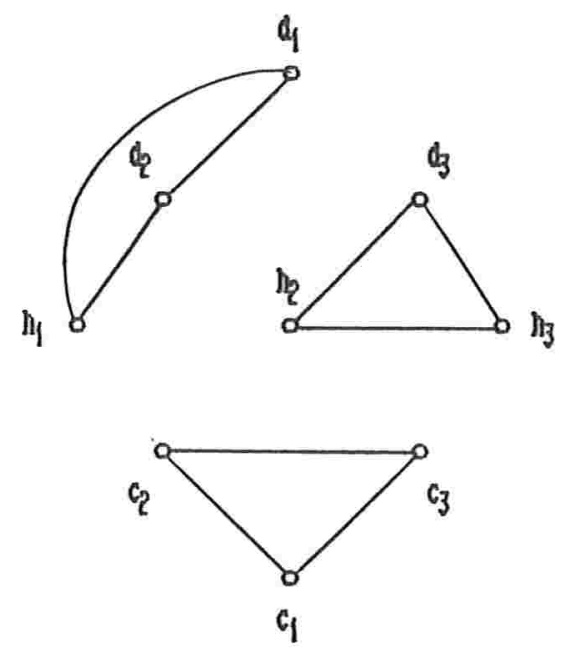

$h_{2}$ adjacente a $d_{3}$
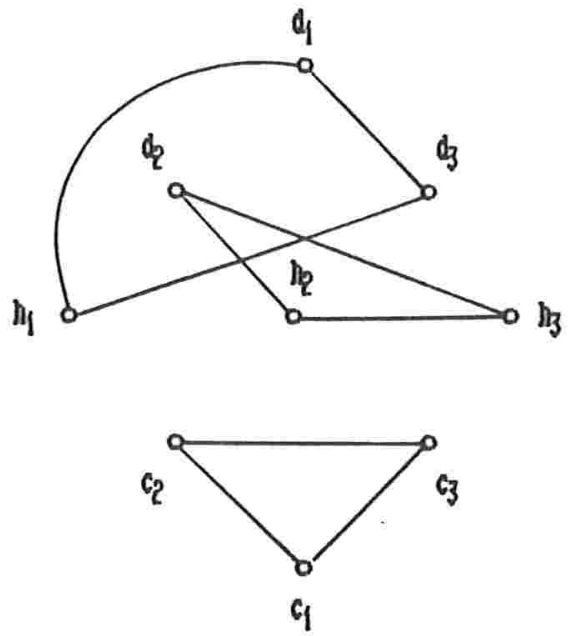

$h_{2}$ adjacente a $d_{2}$

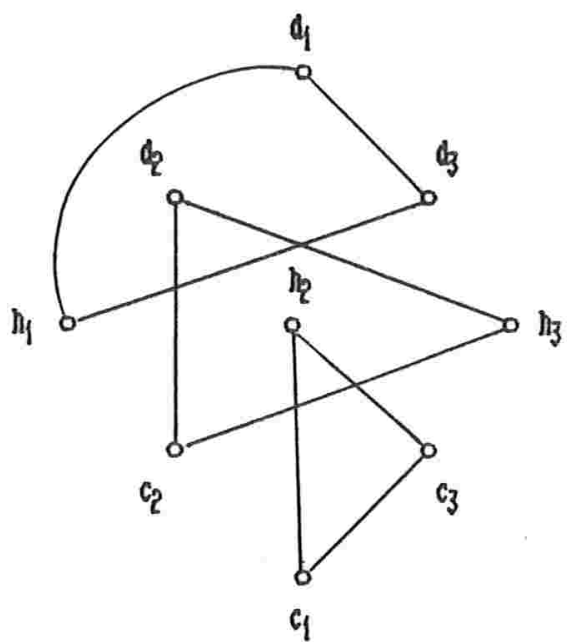

$c_{2}$ adjacente a $d_{2}$

Subcaso 2.2: Nenhuma das 4 arestas indicadas no subcaso anterior está em G.

Seja $S$ o conjunto $\left\{h_{2}, h_{3}, c_{1}, d_{2}\right\}$ e seja $w \circ$ conjunto VG - (VH U VC U VD). Observe que

$$
\begin{aligned}
|A(S, W)| & \geq(8 G-8+3)+(8 G-8+2)+(8 G-8+2)+(8 G-8+2) \\
& >8(K-3) \\
& \geq 8|\mathbb{C}-\{C, D\}| .
\end{aligned}
$$

Portanto existe $E$ em $\mathbb{C}-\{C, D\}$ tal que $|A(S, V E)|>8$. Tem-se que $|A(X, V E)| \geq 3$ para algum $x$ em $S$. 
Caso $\{\mathrm{X}\} \cup$ VE contenha os vértices de um circuito $C^{\prime}$ menor que $\mathrm{E}$, como $\mathrm{VH} U$ VC $U$ VD - $\{\mathrm{x}\}$ contém os vértices de dois triangulos C" e C'" , a colecão (C- $-\{, D, E\}) \quad U\left\{C^{\prime}, C^{\prime \prime}, C^{\prime \prime}\right\}$ satisfaz o objetivo (b) do lema. Note que pela hipotese (*) isto não pode ocorrer se $x=h_{2}$ ou $n_{3}$.

Em caso contrário, note que $E$ é um triangulo, $c_{1} h_{2}$ e $h_{3} d_{2}$ são arestas e $\left|A\left(\left\{c_{1}, h_{2}, h_{3}, d_{2}\right\}, V E\right)\right|>8$. Portanto podemos concluir, usando a proposiç̃̃o 2.4 abaixo, que existem 3 vértices, digamos $x, y, z$, em $\left\{c_{1}, h_{2}, h_{3}, d_{2}\right\}$ tais que $\{x, y, z\} \cup V E$ é união disjunta dos vértices de dois circuitos, digamos $C^{\prime}$ e $C^{\prime \prime}$. Basta agora examinar as quatro possiveis escolnas de $x, y, z$ em $\left\{h_{3}, a_{2}, c_{1}\right.$, $\left.h_{2}\right\}$. As figuras abaixo examinam estes casos e mostram que em cada um deles, (VC $\cup$ VD $\cup \mathrm{VH})-\{\mathrm{x}, \mathrm{y}, \mathrm{z}\}$ é união disjunta dos vértices de 2 circuitos, aigamos $C^{\prime \prime \prime}$ e C'"' . Portanto $(C-\{C, D, E\}) \cup\left\{C^{\prime}\right.$, C", C'", C'"'\} satisfaz o objetivo (a) do lema.
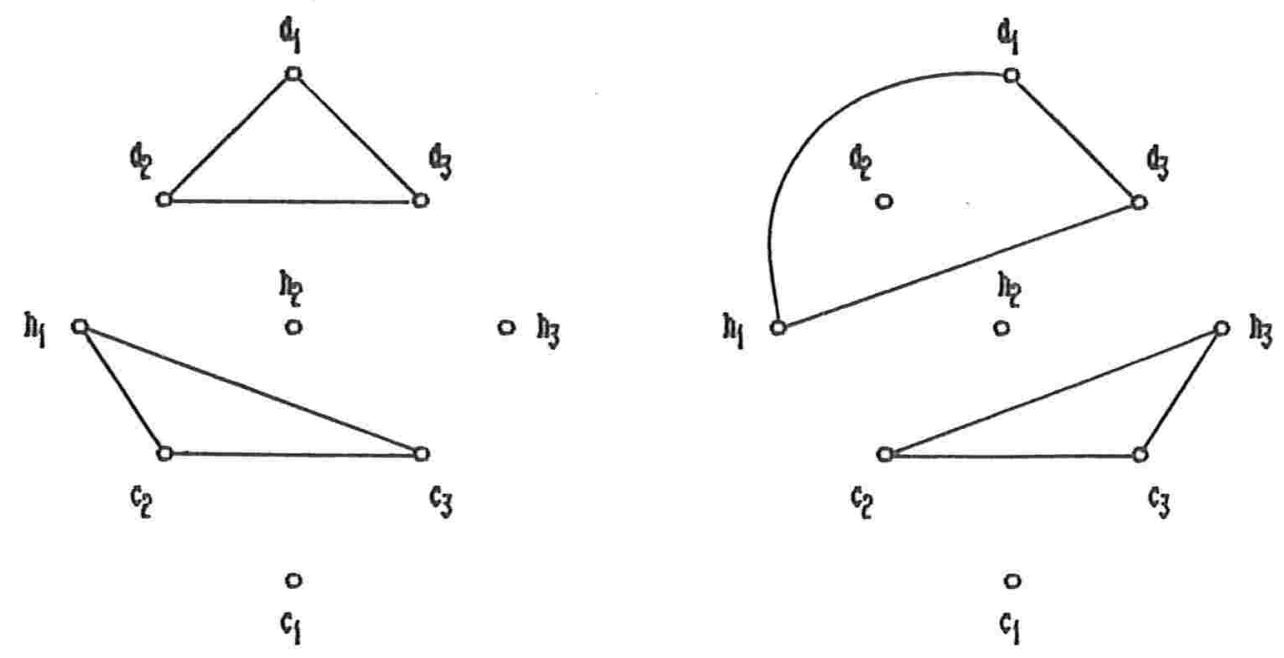

$\{x, y, z\}=\left\{c_{1}, h_{2}, h_{3}\right\}$

$\{x, y, z\}=\left\{c_{1}, h_{2}, d_{2}\right\}$
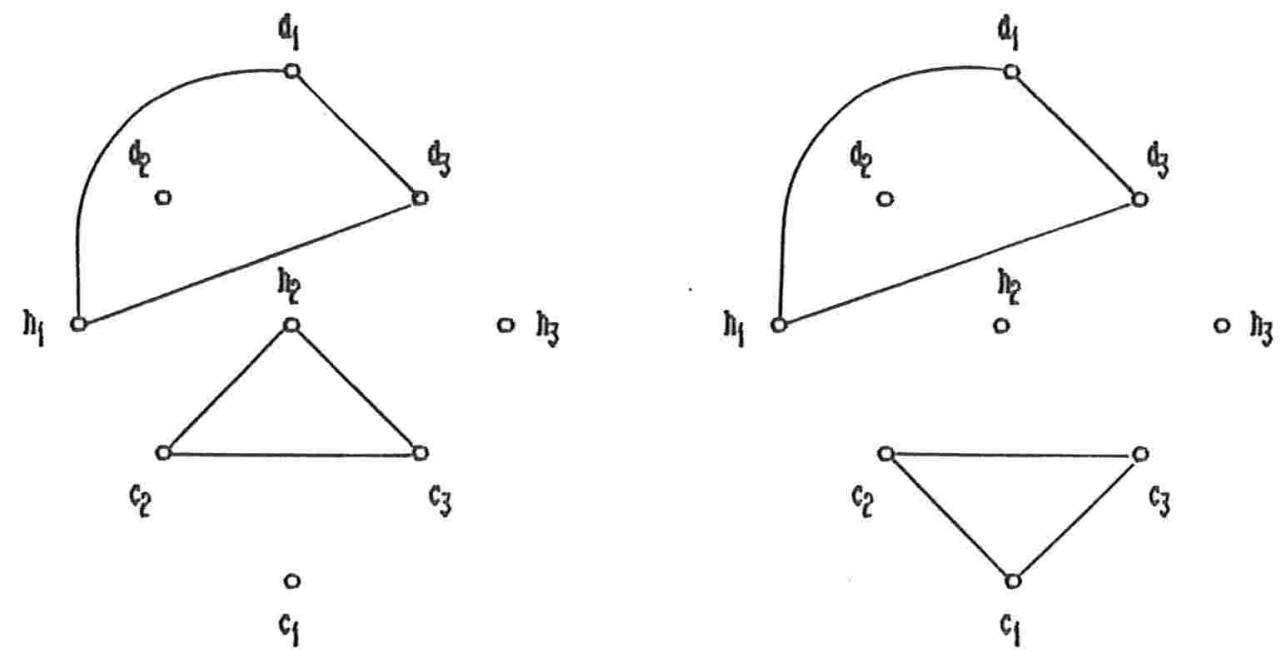

$\{x, y, z\}=\left\{c_{1}, h_{3}, a_{2}\right\}$

$\{x, y, z\}=\left\{h_{2}, h_{3}, d_{2}\right\}$ 
Caso 3: $|V H| \geq 3$ e existe um vertice $h_{O}$ de $H$ com $g_{H}\left(h_{O}\right)=0$.

Seja $h_{1}$ um vértice de $V H-\left\{h_{0}\right\}$ tal que $g_{H}\left(h_{1}\right) \leq 1$. Observe que $\left|A\left(\left\{h_{0}, h_{1}\right\}, V \mathbb{C}\right)\right| \geq \delta G+(\delta G-1)>4(\mathrm{k}-1) \geq 4|\mathbb{C}|$. Logo, existe $\mathrm{C}$ em $\mathbb{C}$ tal que $\left|A\left(\left\{\mathrm{~h}_{0}, \mathrm{~h}_{1}\right\}, \mathrm{VC}\right)\right|>4$. Em virtude da hipótese (*), $\mid \mathrm{VCl}=3$ e uma das duas alternativas vale:

ou

$$
\left|A\left(h_{0}, V C\right)\right|=3 \text { e }\left|A\left(h_{1}, V C\right)\right| \geq 2
$$

$$
\left|\mathrm{A}\left(\mathrm{h}_{\mathrm{O}}, \mathrm{VC}\right)\right| \geq 2 \text { e }\left|\mathrm{A}\left(\mathrm{h}_{1}, \mathrm{VC}\right)\right|=3 .
$$

Em qualquer das duas alternativas existe $c$ em VC tal que (VC - $\{\mathrm{c}\}) \cup\left\{h_{\mathrm{O}}\right\}$ contém os vértices de um circuito $\mathrm{C}^{\prime}$ e $\mathrm{c}$ é adjacente a $n_{1}$.

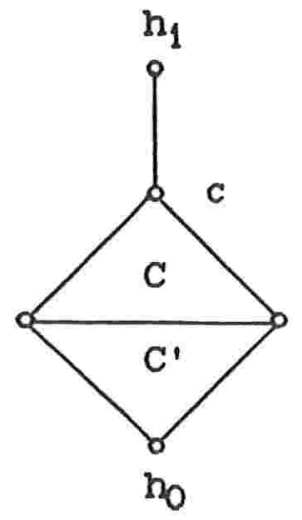

Seja $\mathbb{C}^{\prime}$ a coleção $(\mathbb{C}-\{C\}) \cup\left\{C^{\prime}\right\}$. Observe que $c$ e $h_{1}$ são adjacentes em G - VC' e portanto $\mathbb{C}^{\prime}$ atinge o objetivo (c) do lema.

Caso 4: Para todo vértice $\mathrm{h}$ de $\mathrm{H}, \mathrm{g}_{\mathrm{H}}(\mathrm{h}) \leq 2$; ademais existem pelo menos 4 vértices de $\mathrm{H}$ de grau maior ou igual a $1 \mathrm{em} \mathrm{H}$.

Existem 4 vértices distintos em $\mathrm{H}$, digamos $\mathrm{v}, \mathrm{w}, \mathrm{x}, \mathrm{y}$, tais que

$$
\begin{aligned}
& g_{H}(v)=g_{H}(x)=1, \\
& v \text { adjacente a w e } \\
& x \text { adjacente a } y .
\end{aligned}
$$

Observe que $|A(\{v, w, x, y\}, V)| \geq 2(\delta G-1)+2(\delta G-2)$

$$
\begin{aligned}
& >8(\mathrm{~K}-1) \\
& \geq 8|\mathbb{C}| .
\end{aligned}
$$


Portanto existe $\mathrm{C}$ em $\mathbb{C}$ tal que $|A(\{v, w, x, y\}, v C)|>8$. Então existe um vértice $z$ em $\{v, w, x, y\} \operatorname{com}|A(z, v C)| z 3$, e pela. hipótese (*) temos que $|\mathrm{VC}|=3$. Podemos agora concluir, usando a proposição 2.4, que VH U VC contem os vertices de dois circuitos disjuntos, digamos C' e C" . Basta trocar C por C' e C" em C para obter uma coleção $\mathbf{C}^{\prime}$ que satisfaz o objetivo (a) do lema.

Caso 5: $\mathrm{H}$ tem um vertice $v$ tal que $\mathrm{g}_{\mathrm{H}}(\mathrm{v}) \geq 3$.

Seja $F$ um conjunto de exatamente 3 folhas da componente de $\mathrm{H}$ que contem $\mathrm{v}$. Observe que

$$
|A(F, V \mathbb{C})| \geq 3(\delta G-1)>6(\mathrm{~K}-1) \geq 6|\mathbb{C}| .
$$

Portanto existe $C$ em $\mathbf{C}$ tal que $|A(F, v C)| \geq 7$. Segue-se que existe $\mathrm{x}$ em $\mathrm{F}$ tal que $|\mathrm{A}(\mathrm{x}, \mathrm{VC})| z 3$, donde, pela hipotese (*), $|V C|=3$. Seja $\pi$ um caminho em $H$ ligando os dois vértices de $F-\{x\}$. Note que existe $c$ em $C$ adjacente a ambos os extremos de

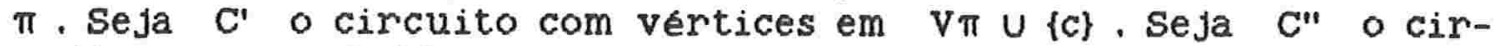
cuito com vértices em (VC - $\{c\}) U\{x\}$. A colecão $(\mathbb{C}-\{C\}) \cup\left\{C^{\prime}, C^{\prime \prime}\right\}$ satisfaz o objetivo (a) do lema.

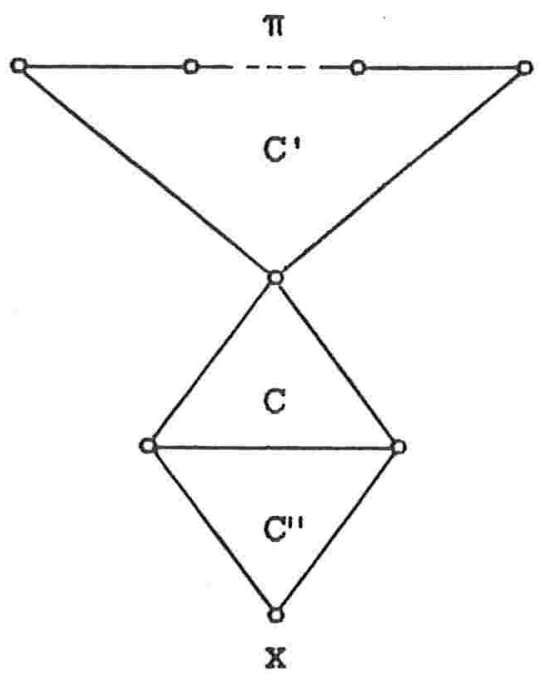

Está terminada a prova do lema, a menos do seguinte:

Proposicão 2.4: Seja $G$ um grafo com um triangulo $C$ e um conjunto de vertices $x=\{x, y, v, w\}$, tais que $V C \cap x=\varnothing$, $|A(X, V C)|>8, \quad x$ adjacente a $y$ e $V$ adjacente a $w$. Então existe um vertice $t$ em $x$ tal que $V C U(X-\{t\})$ eे o conjunto de vertices de dois triangulos dis juntos.

Prova: Iremos mostrar abaixo que existem 3 vértices - digamos p, q, $r-e m\{x, y, v, w\}$ e um verrtice $c$ em $v C$, tais que $p$ é adjacente a $q,|A(c,\{p, q\})|=2$ e $|A(r, V C-\{c\})|=2$. Portanto $\{p, q, r\} \cup V C$ é união disjunta de vértices de dois circuitos, digamos $C^{\prime}$ e C'" 


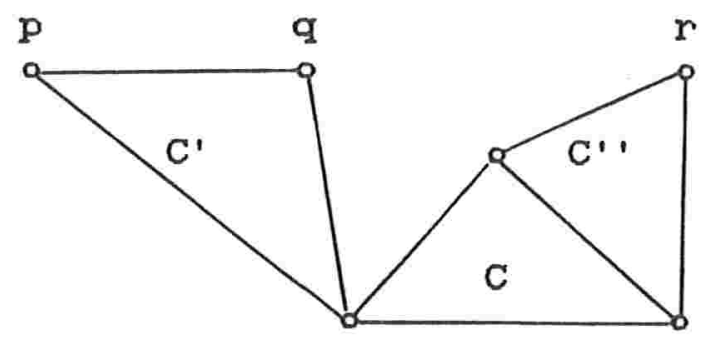

Como $|A(X, V C)|>8$ e $A(t, V C) \leq 3$ para todo $t$ em $x$ temos, a menos de simetria entre o par $x, y$ e o par $v$, w, duas alternativas a analisar.

Suponha que $|A(\{x, y\}, V C)|=5$ e $|A(\{v, w\}, V C)| \geq 4$. Então existe $c$ em $v C$ tal que $|A(c,\{v, w\})|=2$. Como $|A(\{x, y\}, V C)|=5$ existe um vértice em $\{x, y\}$, digamos $x$, tal que $|A(x, v C)|=3$. Neste caso podemos fazer $p=v, q=w e$ $r=x$.

Suponha agora que $|\mathrm{A}(\{\mathrm{x}, \mathrm{y}\}, \mathrm{vC})|=6$ e $|\mathrm{A}(\{\mathrm{v}, \mathrm{w}\}, \mathrm{vC})| \geq 3$. Então existe um vértice em $\{v, w\}$, digamos $v$, tal que $|A(v, V C)| \geq 2$. Se $|A(v, V C)|=2$, então seja $c$ o vértice de $V C$ que não é adjacente a $v$. Se $|A(v, V C)|=3$, escolha qualquer $c$ em VC, Como $|A(\{x, y\}, V C)|=6,|A(\{x, y\}, c)|=2$. Neste caso podemos fazer $p=x, q=y$ e $r=v$, terminando esta demonstração.

\section{Complexidade computacional}

A prova do lema 2.1 induz um algoritmo, que chamaremos de algoritmo 1, que recebe como entrada um grafo $G$ e uma coleção de circuitos $\mathbb{C}$ e fornece como saida uma coleça $\mathbb{C}^{\prime}$ que satisfaz um dos objetivos do lema. Vamos calcular uma delimitação superior do tempo que uma execução desse algoritmo consome, em termos de $n=|V G|$ e $m=|A G|$.

A construção de $\mathrm{H}$ requer tempo $\mathrm{O}(\mathrm{n} \cdot \mathrm{m})$. A determinação de circuitos em H, via busca em largura, gasta $O(n \cdot m)$ no pior caso. Para a verificação da hipotese (*) devemos: tempo $O(n \cdot m)$.

(1) Verificar se os circuitos de $\mathbf{C}$ tem cordas; isto requer

(2) Para cada vértice $\mathrm{h}$ de $\mathrm{H}$ contabilizar o número de vizinhos de $\mathrm{h}$ em cada circuito de $\mathbb{C}$; essa contabilidade leva tempo proporcional a $\mathrm{m}$. Agora a hipotese (*) deve ser verificada para cada um dos circuitos; caso a hirstese não se verifique, poderemos construir, em tempo proporcion?l a $\mathrm{n}$, a nova coleção $\mathbb{C}^{\prime}$.

Para a determinação de qual dos 5 casos se aplica, basta calcular as componentes conexas de $\mathrm{H}$, o que requer tempo $\mathrm{O}(\mathrm{n} \cdot \mathrm{m})$. 

$O(n \cdot m)$.

Concluimos, portanto, que as verificações iniciais requerem tempo

O caso 1 envolve a escolna de um circuito $C$ de comprimento máximo em $\mathbf{C}$; isto consome tempo $O(n)$. A seguir deve ser feita uma distribuiç̃o de arestas entre vertices de $\mathrm{C}$ e circuitos de $\mathbf{C}-\{C\}$, até encontrar o circuito $D$ desejado. O restante das operacões envolvem os vértices dos dois circuitos e a contabilização das arestas de $A(V C, V D)$, requerendo tempo $O(m)$. Resulta portanto que a complexidade do caso 1 é limitada por $O(m)$.

No caso 2 devemos inicialmente fazer uma distribuiço das arestas com uma ponta em VH e outra nos circuitos de $\mathbf{C}$, até encontrarmos $C$ em $C$ tal que $|A(V H, V C)| z 7$. Contabilizando-se no máximo $6|C|+1$ dessas arestas encontra-se o circuito desejado. Como $|\mathbb{C}|<k<n$, isto requer tempo $O(n)$. A seguir possivelmente mais duas vezes se repetem operaços desse tipo (determinação dos circuitos D e E ). O restante do caso requer tempo constante. Assim, a complexidade do caso 2 é limitada por $O(n)$.

Os casos 3 e 4 envolvem basicamente uma distribuicão de arestas entre $\mathrm{VH}$ e VC, requerendo tempo $O(\mathrm{n})$.

O caso 5 se inicia com uma distribuição de arestas entre vH e $V \mathbb{C}, O$ que pode ser feito em tempo $O(n)$. O caminho $\pi$ requer $O(n)$ para ser encontrado. O restante das operacões deste caso requerem tempo constante, o que dá ao caso 5 uma complexidade limitada por $O(n)$.

Conclui-se que cada chamada do algoritmo 1 requer tempo $O(n \cdot m)$.

Agora considere um algoritmo, que chamaremos de algoritmo 2, que recebe $G$ e $\mathbb{C}$ (que satisfazem as condições do enunciado do lema), e devolve uma colesão $\mathbb{C}^{\prime}$, com $|\mathbb{C}|+1$ circuitos. o algoritmo 2 consiste essencialmente em chamadas consecutivas do algoritmo 1, cada chamada sendo alimentada pela coleção de circuitos $\mathbb{C}^{\prime}$ fornecida pela chamada anterior, até atingir o objetivo (a). E necessario saber quantas vezes o algoritmo 1 pode ser usado sucessivamente sem que o objetivo (a) seja atingido. $E$ isso que faremos a seguir.

Os objetivos (b) e (c) não obtem uma coleção de circuitos maior do que $\mathbb{C}$. O objetivo (b) aumenta o numero de vértices em VG - VC , e portanto nāo pode ser atingido mais do que $n$ vezes em chamadas sucessivas do lema. O objetivo (c) aumenta o numero de arestas em $\mathrm{AH}$. Com 3 ou mais arestas em $\mathrm{H}$ ocorre um dos dois seguintes casos do lema: ou para todo vértice $h$ de $H, g_{H}(h) \leq 2$ e existem pelo menos 4 vértices de $H$ de grau maior ou igual a 1 (caso 4) ou $H$ tem um vértice $h$ tal que $g_{H}(h) \quad 23$ (caso 5). A salda desses casos são coleções de tamanho maior que $\mathbb{C}$. O pior caso ocorre, portanto, quando o lema atinge duas vezes consecutivas o objetivo (c) e a seguir atinge o objetivo (b). Essa seqüencia não pode se repetir me is do que $n$ vezes, resultando que no máximo $3 n+1$ vezes $(O(n)$ vez s) o algoritmo 1 pode ser usado sem aumentar o tamanho de $\mathbb{C}$. Resumindo, a coleção $\mathbb{C}$ aumenta apos não mais que $O(n)$ chamadas consecutivas do algoritmo 1. 
Como cada chamada do algoritmo 1 requer tempo $O(n \cdot m)$, resulta que aumentar a cardinalidade da coleção requer tempo total $O\left(n^{2} \cdot m\right)$. Para obter uma coleção com $k$ circuitos disjuntos é necessário repetir esse processo $k$ vezes. Como $k<n$ obtemos uma delimitacão superior total de $\mathrm{O}\left(\mathrm{n}^{3} \cdot \mathrm{m}\right)$. 


\title{
Uma extensão do teorema de
}

\section{Corrádi e Hajnal}

\begin{abstract}
Observando o resultado obtido por Corrádi e Hajnal (apresentado no capitulo anterior), e mais uma extensão do mesmo feita por ele e Erdös na mesma época, Dirac [Di63] comenta: "These results suggest the question, -- which $(2 k-1)$ connected graphs do not contain $k$ mutually disjoint circuits?"; e continua: "This question will here be answered for the case $k=2$, graphs with multiples edges being allowed". Este capitulo responde parcialmente uma generalização da pergunta. Vamos provar o seguinte teorema: para qualquer natural $\mathrm{K} \geq 4$, todo grafo $\mathrm{G}$ tal que $|\mathrm{VG}| \geq 4 \mathrm{~K}, \gamma \mathrm{G} \geq 3$ e $\delta G \geq 2 \mathrm{k}-1$ contém $k$ circuitos disjuntos se e só se $\tau_{2} G \geq K$. Foi visto no capitulo 1 que $T_{2} G \geq \mathrm{K}$ é condição necessaria para a existencia dos $k$ circuitos, restando portanto a demonstração de que a condição, neste caso, também é suficiente.
\end{abstract}

O teorema não cobre os casos $k=1,2,3$. Vamos discutir brevemente estes casos. Trocando " $\mathrm{k} \geq 4$ " por " $\mathrm{k}=1$ " o enunciado do teorema continua sendo uma afirmação verdadeira. Para $\mathrm{k}=2$ obtemos uma afirmação verdadeira desde que $G$ não seja uma roda; isto foi demonstrado por Lovász [Lo65] como já dissemos no cap. 1. Conjecturo que a afirmação continua válida com $\mathrm{k}=3$ no lugar de $\mathrm{k} \geq 4$. A conjectura se baseia no seguinte: A maior parte da demonstração do teorema (para o caso $\mathrm{k} \geq 4$ ) se aplica também ao caso $\mathrm{k}=3$. Há apenas uma afirmação durante a demonstração que poderia ser falsa quando $\mathrm{k}=3$. Para confirmar a veracidade daquela afirmaça no caso $\mathrm{k}=3$, é necessário fazer uma análise exaustiva dos possiveis subgrafos de $G$ que satisfazem certas restrições. Fiz esta análise e acredito que confirmei a validade da afirmação para $k=3$. Os detalhes são, entretanto, por demais cansativos para inclusão neste trabalho.

Consideremos agora o que acontece se a condição |VG| $\geq 4 \mathrm{~K}$ é relaxada, enquanto as demais condições (em particular $k \geq 4$ ) são mantidas. E claro que se $|V G|<3 k$, então $G$ não tem $k$ circuitos disjuntos, pois $\gamma G \geq 3$. Para $3 \mathrm{~K} \leq|\mathrm{VG}|<4 \mathrm{~K}$ não sabemos se a afirmacão do teorema permanece verdadeira. A análise deste caso é complicada pelo mesmo motivo apontado no capitulo anterior, ou seja, é necessário obter $k$ circuitos a partir de $k-1$ circuitos quase todos de comprimento $\leq 4$. 
A titulo de curiosidade constatamos que, dentre os grafos $G$ que satisfazem $\gamma G \geq 3, \delta G \geq 2 k-1,3 K \leq|V G|<4 k$ e $\tau_{2} G \geq k$ para $k=3$, somente o grafo da figura abaixo não tem 3 circuitos disjuntos.

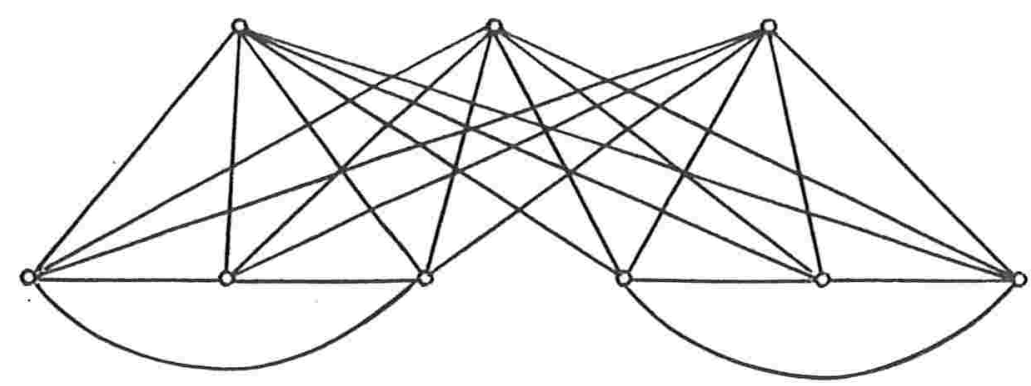

Este grafo tem tambem a peculiar propriedade de que $\tau_{q} \geqslant 3$ para todo $q$, como mostramos na proposição A.3 do Apendice.

\section{O Teorema}

Teorema 3.1: Para qualquer inteiro $k \geq 4$, todo grafo $G$ tal que $|V G|>4 K, \gamma G \geq 3$ e $\delta G \geq 2 K-1$ tem $k$ circuitos disjuntos ou uma 2-transversal de tamanho menor que $k$.

Analogamente à demonstração do teorema 2.1, este teorema pode ser reduzido ao seguinte lema:

Lema 3.1: Seja $K$ um inteiro 24 . Para qualquer grafo $G$ tal que $|V G| \geq 4 K, \gamma G \geq 3$ e $\delta G \geq 2 K-1$ e para qualquer colecão $\mathbb{C}$ de circuitos disjuntos, se $|\mathbb{C}|<k$ então uma das af irmacōes abaixo é verdadeira:

(1) existe em $G$ uma 2 -transversal de tamanho $k-1 / 2$;

(2) existe uma colecz̃o $\boldsymbol{C}^{\prime}$ de circuitos disjuntos nos vertices tal que (2a) $\left|C^{\prime}\right|>|\mathbb{C}|$

ou $(2 \mathrm{~b})\left|\mathbb{C}^{\prime}\right|=|\mathbb{C}|$ mas $\left|\mathrm{VH}^{\prime}\right|>|\mathrm{VH}|$

ou (EC) $\left|\mathbb{C}^{\prime}\right|=|\mathbb{C}|$ e $\left|V^{\prime}\right|=|V H| \operatorname{mas}\left|A H^{\prime}\right|>|A H|$, ande $H=G-V$ e $H^{\prime}=G-\mathbb{C}^{\prime}$.

Repetindo o esquema do capitulo anterior, este lema sugere um processo iterativo para que seja atingido ou o objetivo (1) ou o objetivr (2a). O processo começa com o grafo G e uma coleção de circuitos (possivelmente vazia); usa-se o lema repetidas vezes, cada vez ( )meçando com a coleção $\mathbb{C}^{\prime}$ fornecida pela chamada anterior. As alternativas (2b) e (2C) não podem se repetir indefinidamente. Após um número finito de iterações, atinge-se o objetivo (1) ou o (2a). Este processo prova o teorema a partir do lema. 
Prova do Lema : A prova do lema terá um caráter algoritmico e portanto vamos nos referir a (1), (2a), (2b) e (2c) como "objetivos" do lema. Podemos supor, a exemplo do que foi feito no lema 2.1, que

(i) $\mathrm{H}$ é uma floresta,

(ii) os circuitos de $\mathbb{C}$ não têm cordas $e$

(iii) a hipótese (*) é válida, ou seja, para todo C em $\mathbb{C} e$ todo $h$ em $V H$ pelo menos uma das seguintes afirmações é verdadeira:
$|\mathrm{A}(\mathrm{h}, \mathrm{VC})| \leq 1 ;$
(*) C é um triangulo;
C é um quadrado, $|A(h, v C)|=2$ e h não é adjacente
a vértices consecutivos de $C$.

A não validade de (i) implica em se atingir imediatamente o objetivo (2a). Com a não validade de (ii) ou (iii) o objetivo (2b) é atingido. A prova se desdobra então em 4 casos, dependendo do tamanho de $\mathrm{H}$ e do tamanho de suas componentes.

Caso 1: $|\mathrm{VH}|<4$.

Seja C um circuito de $\mathbf{C}$ tal que $|\mathrm{VC}|$ é máximo. Como

$$
|\mathrm{VC}|=|\mathrm{VG}|-|\mathrm{VH}| \geq 4 \mathrm{~K}-3>4(\mathrm{k}-1) \geq 4|\mathbb{C}|
$$

devemos ter $|\mathrm{VC}|>4$. Como $\mathrm{C}$ não tem cordas, vale $|\mathrm{A}(\mathrm{C}, \mathrm{VG}-\mathrm{VC})|$ $\geq \delta G-2 \geq 2 k-3$ para cada $c$ em VC, donde

$$
|A(V C, V G-V C)| \geq|V C| \cdot(2 k-3) \text {. }
$$

Por outro lado, $|A(V C, h)| \leq 1$ para cada $h$ em $V H$, em virtude da hipotese (*). Segue-se que

$$
\begin{aligned}
|A(V C, V C-V C)| & \geq|V C| \cdot(2 k-3)-|V H| \\
& \geq|V C| \cdot(2 k-3)-3 \\
& >2|V C| \cdot(k-2) \\
& \geq 2|V C| \cdot|C-\{C\}| .
\end{aligned}
$$

Portanto existe $D$ em $\mathbb{C}-\{C\}$ tal que $|\mathrm{A}(\mathrm{VC}, \mathrm{VD})| \geq 2|\mathrm{VC}|+1$. Pela maximalidade de $C$ vale também $|A(V C, V D)| \geq 2|V D|+1$.

Podemos agora usar o mesmo esquema do caso 1, lema 2.1, para encontrar dois circuitos disjuntos, $C^{\prime}$ e $D^{\prime}$, com vértices em VC $U$ VD, tais que $\left|V C^{\prime}\right| \leq|V C|$ e $\left|V D^{\prime}\right| \leq|V D|$, com uma das desigualdades sendo estrita. Trocando-se $C$ e $D$ por $C^{\prime} e D^{\prime}$ em $\mathbb{C}$, obtem-se uma nova coleção de circuitos que cumpre o objetivo (2b) do rema.

Caso 2: $|\mathrm{VH}| \geq 4$ e existe $\mathrm{h}_{\mathrm{O}}$ em vH tal que $g_{\mathrm{H}}\left(\mathrm{h}_{\mathrm{O}}\right)=0$.

Seja $h_{1}$ um vértice de $V H-\left\{h_{0}\right\}$ tal que $g_{H}\left(h_{1}\right) \leq 1$. Observe que $\left|A\left(\left\{h_{O}, h_{1}\right\}, V \mathbb{C}\right)\right| \geq(\delta G-1)+(\delta G-2)>4(\mathrm{k}-1) \geq 4|\mathbb{C}|$. 
Logo, existe $\mathrm{C}$ em $\mathbb{C}$ tal que $\left|\mathrm{A}\left(\left\{\mathrm{h}_{\mathrm{O}}, \mathrm{h}_{1}\right\}, \mathrm{VC}\right)\right|>4$. Pela hipotese (*), $|\mathrm{VC}|=3$ e uma das duas alternativas vale:

ou

$$
\begin{aligned}
& \left|A\left(h_{0}, V C\right)\right|=3 \text { e }\left|A\left(h_{1}, V C\right)\right| \geq 2 \\
& \left|A\left(h_{O}, V C\right)\right| \geq 2 \text { e }\left|A\left(h_{1}, V C\right)\right|=3 .
\end{aligned}
$$

Em qualquer das duas alternativas existe $c$ em vC tal que (VC - $\{\mathrm{C}\}$ ) $\cup\left\{\mathrm{h}_{\mathrm{O}}\right\}$ contém os vértices de um circuito $\mathrm{C}^{\prime} \mathrm{e}$ c é adjacente a $h_{1}$. Seja $\mathbb{C}^{\prime}$ a coleção $(\mathbb{C}-\{C\}) \cup\left\{C^{\prime}\right\}$. Observe que $c$ e $h_{1}$ são adjacentes em $G-V C^{\prime}$ e portanto $C^{\prime}$ atinge o objetivo (2c) do lema.

Caso 3: $|\mathrm{VH}| \geq 4, \mathbf{g}_{H}(\mathrm{~h}) \geq 1$ para todo $\mathrm{h}$ em VH, mas $\mathrm{H}$ não tem duas arestas disjuntas nos vértices.

Então $H$ é uma estrela, isto é, tem a forma descrita na figura abaixo. $H$ tem um unico vértice, $v$, que não é folha; todos os demais vértices são folhas e são adjacentes a $v$.

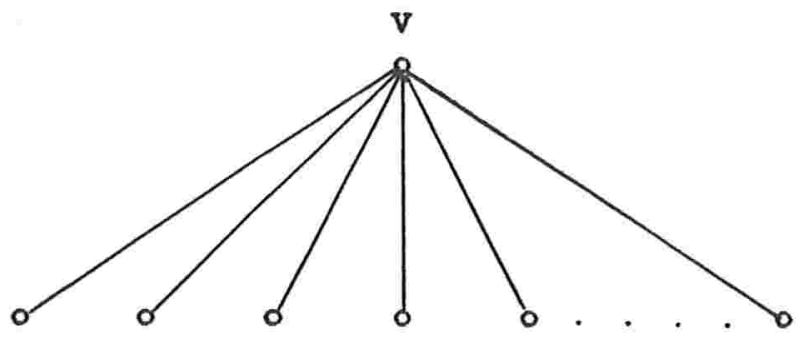

Seja X o conjunto de folhas de H. Vamos procurar encontrar uma das duas configurações seguintes:

(1) $\mathrm{x} e \mathrm{~m} x, \mathrm{C}$ em $\mathbb{C}$ e $\mathrm{c}$ em vC tais que $|A(x, V C-\{c\})| \geq 2$ e $|A(C, X-\{x\})| \geq 2$,

ou (2) $x \mathrm{em} x, C \mathrm{C}$ D em $\mathbb{C}, c$ em VC, $d \mathrm{em}$ VD tais que $|A(x, V C-\{c\})| \geq 2,|A(d, x-\{x\})| \geq 2 e$ $|A(C, V D-\{d\})| \geq 2$.
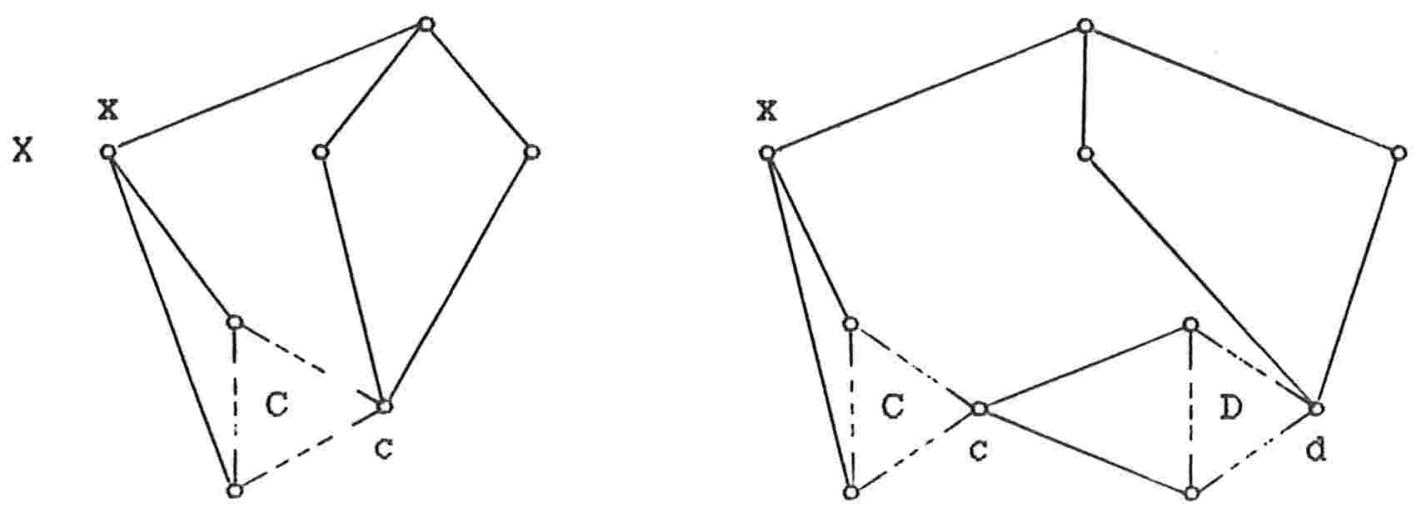

Qualquer destas configurações leva imediatamente à consecução do objetivo (2a). 
Subcaso 3.1: Existe $\mathrm{C}$ em $\mathbb{C}$ tal que $|\mathrm{A}(\mathrm{X}, \mathrm{VC})|>2|X|$.

Neste caso existe $\mathrm{x}$ em $\mathrm{x}$ tal que $|\mathrm{A}(\mathrm{x}, \mathrm{VC})|>2$. Pela $\mathrm{hi}-$ pótese (*), C é um triângulo e $|A(x, V C)|=3$. Por outro lado, como $|\mathrm{X}| \geq 3$, tem-se $|\mathrm{A}(\mathrm{VC}, \mathrm{X}-\{\mathrm{X}\})|>3$. Portanto existe $\mathrm{c}$ em $\mathrm{C}$ tal que $|A(c, X-\{X\})| \geq 2$, obtendo-se assim a configuração desejada.

Subcaso 3.2: $|A(X, V C)| \leq 2|X|$ para cada $C$ em $\mathbb{C}$, mas existem $\mathrm{x}$ em $\mathrm{X}$ e $\mathrm{C}$ em $\mathbb{C}$ tais que $|\mathrm{A}(\mathrm{x}, \mathrm{vC})|>2$.

Pela hipotese (*), $|\mathrm{VC}|=3$. Como $|\mathrm{A}(\mathrm{X}, \mathrm{V} \mathbb{C})| \geq(\delta \mathrm{G}-1) \cdot|\mathrm{X}|$ $=2(\mathrm{~K}-1) \cdot|\mathrm{X}| \geq 2|\mathbb{C}| \cdot|\mathrm{X}|$ temos tambem que $|\mathrm{A}(\mathrm{X}, \mathrm{VC})|=2|\mathrm{X}|$. Por outro lado, para qualquer $D$ em $\mathbb{C}$, existe $y$ em $X$ tal que $|A(Y, V D)| \geq 2$, donde, pela hipotese (*), $|V D| \leq 4$. Logo $|X|$

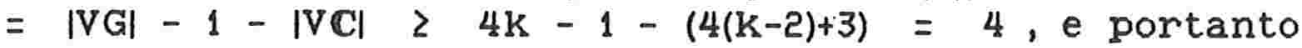

$$
\begin{gathered}
|A(X, V C)| \geq 8 \text { e } \\
|A(V C, X-\{x\})|=|A(V C, X)|-|A(V C, x)| \geq 8-3=5 .
\end{gathered}
$$

Logo existe $c$ em $C$ tal que $|A(c, X-\{x\})| \geq 2$, obtendo-se assim a configuração desejada.

Subcaso 3.3: Para cada $\mathrm{x}$ em $\mathrm{x}$ e cada $\mathrm{C}$ em $\mathbb{C},|\mathbb{A}(\mathrm{x}, \mathrm{VC})| \leq 2$.

Então, como no subcaso anterior, $|A(X, D)|=2|X|$ para cada $D$ em $\mathbb{C} e|A(x, D)|=2$ para cada $x$ em $X$ e cada $D$ em $\mathbb{C}$.

Alternativa 3.3.1: Existem $x_{1}$ e $x_{2}$ em $x$ e $C$ em $\mathbb{C}$ tais que os vizinhos de $x_{1} \quad e \quad x_{2}$ em $C$ não são os mesmos.

Seja $c_{1}$ um vértice de $C$ adjacente a $x_{1}$ mas não a $x_{2}$. Defina $c_{2}$ analogamente trocando $x_{1}$ por $x_{2}$. Pela hipotese (*), $|\mathrm{VC}| \leq 4$ e $c_{1}$ e $c_{2}$ são consecutivos em C . Como $|\mathrm{X}| \geq 3$, podemos garantir que existem i e $j$ distintos tais que

$$
\left|A\left(x_{j}, V C-\left\{C_{i}\right\}\right)\right| \geq 2 \text { e }\left|A\left(c_{i}, X-\left\{x_{j}\right\}\right)\right| \geq 2,
$$

obtendo-se assim a configuração desejada.

Alternativa 3.3.2: Para todo par $x_{1}$ e $x_{2}$ em $x$ e todo $C$ em $\mathbb{C}$, os vizinhos de $\mathrm{x}_{1}$ e $\mathrm{x}_{2}$ em $\mathrm{C}$ coincidem.

Seja $K=\{c \in V \mathbb{C}: c$ é adjacente a $X\}$. Seja $V$ o vertice de $H$ que não é folha. Se toda aresta de $G$ tem uma ponta em $\mathrm{K} U\{\mathrm{v}\}$ então conseguimos o objetivo (1): a transversal que associa valor $1 / 2$ a cada vértice de $\mathrm{K} \cup\{\mathrm{v}\}$ e valor $\mathrm{O}$ aos demais vértices tem tamanho $(2 \mathrm{k}-1) / 2$.

Suponha então que nem toda aresta le $\mathrm{G}$ tem ponta em $\mathrm{K} U\{\mathrm{v}\}$. Seja a uma tal aresta. Ambas as pontas de a estão em VC. Seja c uma das pontas de a e seja $\mathrm{C}$ o elemento de $\mathbb{C}$ que contém $\mathrm{C}$. Escolha $\mathrm{D}$ em $\mathbb{C}-\{\mathrm{C}\}$ da seguinte maneira: se existe $\mathrm{D}$ em $\mathbb{C}-\{\mathrm{C}\}$ tal que $|A(C,-V D)| \geq 3$ então escolha este $D$; senão seja $D$ o circuito que passa pela outra ponta de a e observe que 


$$
|A(C, V C-V C)| \geq \delta G-3=2 k-4 \geq 2|\mathbb{C}-\{C\}|,
$$

donde $|A(C, V D)|=2$. Em qualquer caso, existe aresta com pontas em $c$ e VD, cuja ponta em VD não está em $K$. Agora é fácil ver que existe $c^{\prime}$ em VC, $x$ em $x, d e m$ VD tais que

$\left|A\left(x, V C-\left\{C^{\prime}\right\}\right)\right| \geq 2,|A(d, X-\{x\})| \geq 2,\left|A\left(C^{\prime}, V D-\{d\}\right)\right| \geq 2$

obtendo-se assim a configuração desejada.

Caso 4: $|\mathrm{VH}| \geq 4$ e $\mathrm{H}$ tem quas arestas disjuntas nos vértices.

Sejam $P$ e $Q$ dois caminhos em $H$ maximais em relacão à seguinte propriedade: $P$ e $Q$ são disjuntos nos vértices e cada um deles usa uma das arestas mencionadas acima. Seja $v$ um dos extremos de $P$. Un dos vizinhos de $v$ está em $P$. Se $v$ tiver outros vizinhos em $H$, eles estão todos em VQ. Neste caso $v$ tem somente um vizinho em $V Q$, pois não existem circuitos em $H$. Por esse mesmo motivo podemos concluir que somente um dos extremos de $\mathrm{P}$ pode ter vizinhos em $V Q$, e conseqüentemente un dos extremos de $P$ é uma folha em $H$. O mesmo raciocinio vale para os extremos de $Q$.

Seja $v$ um dos extremos de $P$. Se $v$ é follha de $H$ temos $|\mathrm{A}(\mathrm{v}, \mathrm{VC})| \geq \delta G-1=2 \mathrm{k}-2$. Se $\mathrm{v}$ não é follha de $\mathrm{H}, \mathrm{v}$ tem somente dois vizinhos em $\mathrm{H}$, e portanto $|\mathrm{A}(\mathrm{v}, \mathrm{VC})| \mathrm{Z} \delta \mathrm{G}-2$ $=2 \mathrm{k}-3$. Sejam $\mathrm{X}$ e $\mathrm{Y}$ os conjuntos de vértices que são extremos de $P$ e $Q$, respectivamente. Pelo observado acima temos que $|A(X, V C)| \geq 2 K-2+2 K-3$, O mesmo valendo para $Y$. Resulta então que

$$
|\mathrm{A}(\mathrm{XUY}, \mathrm{VC})| \geq 2(2 \mathrm{~K}-2)+2(2 \mathrm{~K}-3)=8 \mathrm{k}-10>7(\mathrm{k}-1) .
$$

Garante-se assim a existência de um circuito $\mathrm{C}$ de $\mathbb{C}$ tal que $|\mathrm{A}(\mathrm{X} \cup \mathrm{Y}, \mathrm{VC})| \geq 8$. Observe que para concluir que $8 \mathrm{k}-10>7(\mathrm{k}-1)$ precisamos de $\mathrm{k} \geq 4$. Aqui a prova falharia se a hipótese do teorema permitisse $\mathrm{k}=3$.

Com a configuração definida acima, pelo menos um dos vértices de $X \cup Y$ tem duas ou mais arestas com pontas em $C$, o que garante, pela hipótese (*), que $\mathrm{C}$ é triângulo ou quadrado.

Agora não é dificil encontrar um conjunto $Z$ de vértices consecutivos de VC tal que $|A(X, Z)| \geq 2$ e $|A(Y, V C-Z)| \geq 2$. Portanto existe um circuito $C^{\prime}$ com vértices em VP $U Z$, e um circuito $C^{\prime \prime}$ com vértices em $V Q U(V C-Z)$. Trocando-se $C$ por $C^{\prime}$ e $C^{\prime \prime}$ em $\mathbb{C}$, obtem-se uma nova coleção de circuitos que cumpre o objetivo (2a) do lema.

Com isto está terminada a prova do lema. 


\section{Complexidade computacional}

A prova do lema 3.1 incuz um algoritmo, que chamaremos de algoritmo 1, que recebe como entrada um grafo $G$ e uma coleção de circuitos $\mathbb{C}$ e formece como saida uma coleção $\mathbb{C}^{\prime}$ que satisfaz um dos objetivos do lema. Vamos calcular uma delimitacão superior do tempo que uma execução desse algoritmo consome, em termos de $n=|V G| e$ $m=|A G|$.

Analogamente ao algoritmo do lema 2.1, as verificações iniciais (construção de $\mathrm{H}$, determinacão de circuitos, verificação da hipótese (*), decisão de qual dos casos.se aplica) requerem tempo $O(n \cdot m)$.

o caso 1 equivale ao caso 1 do lema 2.1 , e requer tempo $O(m)$. $O$ caso 2 equivale ao caso 3 do lema 2.1 , e requer tempo $O(n)$.

No caso 3 devemos verificar como estão distribuidas as arestas de $\mathrm{A}(\mathrm{VH}, \mathrm{VC})$. Possivelmente essa verificação também é feita para as arestas de $\mathrm{A}(\mathrm{VC}, \mathrm{VC}-\mathrm{VC})$. Isso requer tempo $\mathrm{O}(\mathrm{m})$. O restante das verificaçōes requer tempo constante, resultando para este caso tempo $\mathrm{O}(\mathrm{m})$.

- caso 4 começa com a construção de dois caminhos em $H$, o que requer tempo $O(n)$. Em seguida é feita uma verificaça de como se distribuem as arestas com uma ponta nos extremos dos caminhos e outra $\mathrm{em} \mathrm{VC}$, até encontrar-se o circuito $\mathrm{C}$ procurado, requerendo tempo $O(n)$. Em tempo constante podemos fazer o restante da verificações, $O$ que resulta para esta caso tempo $O(n)$.

Conclui-se que cada chamada do algoritmo 1 requer tempo $O(n \cdot m)$.

Agora considere um algoritmo, que chamaremos de algoritmo 2 , que recebe $G$ e $\mathbb{C}$ (que satisfazem as condições do enunciado do lema), e devolve uma coleção $\mathbb{C}^{\prime}$, com $|\mathbb{C}|+1$ circuitos ou uma 2-tranversal de tamanho $<\mathrm{k}$. $\mathrm{O}$ algoritmo 2 consiste essencialmente em chamadas consecutivas do algoritmo 1,' cada chamada sendo al imentada pela coleção de circuitos $\mathbb{C}^{\prime}$ formecida pela chamada anterior, até atingir o objetivo (1) ou (2a). E necessário saber quantas vezes o algoritmo 1 pode ser usado sucessivamente sem que o objetivo (1) ou (2a) sejam atingidos. E isso que faremos a seguir.

o objetivo (2C) não pode ser atingido mais do que $|\mathrm{VH}|<\mathrm{n}$ vezes consecutivas sem que ocorra ou o objetivo (1) ou o (2a), pois todo grafo com o número de arestas 2 ao número de vértices tem um circuito. Obviamente o objetivo ( $2 b$ ) não pode ser atingido mais do que $n$ vezes sem que se ocorra ou o objetivo (1) ou o (2a). Combinando-se então as possibilidades acima, concluimos que apos $O\left(n^{2}\right)$ chamadas do algoritmo 1 o objetivo (1) ou o (2a) é atingido pelo algoritmo 2. Resulta que cada chamada do algoritmo 2 requer tempo $O\left(\mathrm{n}^{3} \cdot \mathrm{m}\right)$. 
Um algoritmo que receba como entrada $G$ e $k$ e, usando o algoritmo 2 , devolve uma coleção de $k$ circuitos disjuntos ou uma 2 -transversal de tamanho $<\mathrm{k}$ precisa usar o algoritmo 2 no máximo $\mathrm{k}$ vezes, Como $k<n$, obtemos uma delimitacão superior total de $\mathrm{O}\left(\mathrm{n}^{4} \cdot \mathrm{m}\right)$.

Podemos, entretanto, melhorar o tempo estimado do algoritmo 2 . Observe que, apos $|\mathrm{VH}|>4$, saidas do tipo (2b) só são formecidas via verificações iniciais. Nelas é verificado se algum circuito de $\mathbb{C}$ tem corda e são verificadas as condições da hipotese (*). No caso de algum circuito ter corda, e fácil ver que $|\mathrm{AH}|+|\mathrm{VH}|<\mid \mathrm{AH} H^{\prime}+$ $|V H '|$. Observe que a hipotese $(*)$ so é aplicada no decorrer da prova a vértices de $H$ que tem grau menor que 3 . Se o algoritmo 2 se $1 \mathrm{imi-}$ tar a verificar a hipotese $(*)$ a esses vértices de $H$, temos que cada vez que o objetivo (2b) é atingido via verificação da hipotese (*) $|\mathrm{AH}|+|\mathrm{VH}| \leq|\mathrm{AH} \cdot|+\left|\mathrm{VH}^{\prime}\right|$. E bbvio que quando se atinge o objetivo (2C) temos tamberm $|\mathrm{AH}|+|\mathrm{VH}|<\left|\mathrm{AH} H^{\prime}\right|+\left|\mathrm{VH}^{\prime}\right|$, Resumindo: um algoritmo com essa otimização ou formece saidas do tipo (1) ou (2a), ou saidas do tipo (20) e (2c) com $|\mathrm{AH}|+|\mathrm{VH}| \leq|\mathrm{AH} \cdot|+\left|\mathrm{VH}^{\prime}\right|$. Essa desigualdade so pode não ser estrita quando $|\mathrm{VH}|<|\mathrm{VH}|$, o que não pode ocomer mais do que $n$ vezes. Portanto, no máximo em $2 n+1$ $(O(n))$ chamadas do algoritmo 1, o algoritmo 2 nos formece uma saida do tipo (1) ou (2a). Com isso se melhora o tempo total para $O\left(n^{3} \cdot m\right)$. 
Nesta parte do trabalho estão as demonstrações de algumas proposições citadas no decorrer da dissertação. A proposição A.1 é baseada em resultados apresentados por Lovász em " Combinatorial problems and exercises" [Lo79], principalmente nos exercicios 10.16, 10.17 e 10.18. Em geral sabemos que qualquer grafo $G$ não tem mais do que $T G$ circuitos disjuntos, onde $T G$ é a cardinalidade minima de uma transversal de circuitos. Na proposição A.1 se prova que todo grafo G tem uma coleção com pelo menos $\left\lceil\tau \mathrm{G} /\left(3+2 \log _{2}(\tau \mathrm{G})\right)\right\rceil$ circuitos disjuntos. Esse resultado é de certa forma semelhante ao de Erdös e P6sa, citado no capitulo 1, que estabelece que uma condição suficiente para um grafo $G$ ter $K$ circuitos disjuntos é TG $\geq$ c.K. $\log (K)$. A demonstração da proposição é essencialmente um algoritmo que a cada iteração seleciona o menor circuito disponivel. Já mencionamos esse algoritmo no capitulo 1.

A proposição A.2 constrói para cada natural q um grafo G tal que $\tau_{q} G \geq 2$, mas $G$ não tem dois circuitos disjuntos. A proposição A.3 apresenta um grafo que não tem 3 circuitos disjuntos mas satisfaz $\tau_{q} \geq 3$, qualquer que seja o valor de $q$.

A proposição A.4 estabelece que o PCODV é IP-Completo. A demonstração da proposição é basicamente a mesma usada por Garey e Johnson em "Computers and Intractability" [GJ79, teorema 3.7, pág. 68].

A proposicão A.5 mostra que o PCODV e o PCODA, são problemas mutuamente redutiveis em tempo polinomial, provando indiretamente que - PCODA é NP-Completo. As idéias dessa demonstração foram extraidas de [PR78], onde é feita uma demonstração análoga.

Erdös e Posa provaram [EP62] que para qualquer inteiro $k \geq 1$ todo grafo $\mathrm{G}$ com $\gamma \mathrm{gG} 3,|\mathrm{VG}| \geq 24 \mathrm{~K}$ e $|\mathrm{AG}| \geq(2 \mathrm{~K}-1) \cdot|\mathrm{VG}|$ $-2 k^{2}+k+1$, tem $k$ circuitos disjuntos. Erdös indaga [Er71] sobre uma possivel expressão para |AG| sem a restrição ao número de vértices. A proposição A.6 responde de forma satisfatória a esse comentário, obtendo-se $|A G| \geq(2 k-1) \cdot|V G|-3\left(k^{2}+k\right) / 2+4$ e $|V G| \geq 3 k$. A prova da proposição depende do teorema 2.1 e de um teorema de Lovász já citado [L065]. Dirac e Justesen [Di76] têm um resultado ainda melhor: $|A G| 2 \max \{(3 k-1) \cdot(3 k-2) / 2+|V G|-3 k+3$, $\left.(2 \mathrm{~K}-1) \cdot|\mathrm{VG}|-2 \mathrm{~K}^{2}+\mathrm{K}+1\right\}$, para $3 \mathrm{~K} \leq|\mathrm{VG}|<24 \mathrm{~K}$.

A proposição A.7 é usada na base da indução da proposição A.6. O resultado é mencionado por Erdös e Pósa em [EP62] ccmo um trabalho não publicado de Dirac. 
Proposicão A.1: Em todo grafo $G$ tal que $T G \geq 1$ existem pelo menos $\Gamma \tau G /(3+21082(T G)) 7$ circuitos disjuntos.

Prova: Vamos fazer a prova por indução em $T G$. Se $T G \leq 9$ a proposiç̃o é trivialmente válida, pois $\left\lceil 9 /\left(3+2 \log _{2}(9)\right)\right\rceil=1$.

Supondo $T G>9$, devemos inicialmente obter a partir de $G$ um grafo $\mathrm{G}_{\mathrm{a}}$. Começamos com $\mathrm{G}_{\mathrm{a}}=\mathrm{G}$, e repetimos as operações (i) $\mathrm{e}$ (ii) abaixo até que $\delta G_{a} \geq 3$ ou até $\gamma G_{a}=2$.

(i) Se $G_{a}$ tem um vértice $v$ com graus 1 , então faça $\mathrm{G}_{\mathrm{a}}=\mathrm{G}_{\mathrm{a}}-\{\mathrm{v}\} ;$

(ii) Se $G_{a}$ tem um verrtice $v$ de grau 2 , então sejam $v_{1} e$ $v_{2}$ os vizinhos de $v$. Faca $G_{a}=G_{a}-\{v\}$ e acrescente em $A_{a}$ uma aresta ligando $v_{1}$ a $v_{2}$.

Com essas operações é fácil ver que $T \mathrm{G}_{\mathrm{a}}=\mathrm{TG}$ e a toda coleção de circuitos disjuntos em $G$ corresponde uma coleção de circuitos disjuntos em $G_{a}$, e vice-versa. Resta pois provar que existem pelo menos $\left\lceil\tau G_{a} /\left(3+2 \log _{2}\left(\tau G_{a}\right)\right)\right\rceil$ circuitos disjuntos em $G_{a}$.

Seja $\mathrm{C}$ um circuito de $\mathrm{G}_{\mathrm{a}}$ de comprimento minimo. Se $\delta \mathrm{G}_{\mathrm{a}} \geq 3$ então iremos usar usar aqui o seguinte resultado que se encontra no livro de Lovász [Lo79, exercicio 10.16]: em todo grafo G com $\delta G \geq 3$,

$$
T \mathrm{G} 23 / 8 \cdot 2^{\gamma \mathrm{G} / 2} \text {. }
$$

Dessa expressão podemos deduzir que

$$
\gamma \mathrm{G}_{\mathrm{a}} \leq 3+2 \log _{2}\left(\tau \mathrm{G}_{\mathrm{a}}\right) \text {. }
$$

Se $\delta G_{\mathrm{a}}<3$ então $\gamma \mathrm{G}_{\mathrm{a}}=2$ e também vale a desigualdade acima.

Seja $G^{\prime}=G_{a}-V C$. Claramente

e portanto

$$
\tau \mathrm{G}_{\mathrm{a}} \leq \tau \mathrm{G}^{\prime}+\gamma \mathrm{G}_{\mathrm{a}},
$$

$$
T G^{\prime} \geq T G_{a}-\left(3+2 \log _{2}\left(T G_{a}\right)\right) \text {. }
$$

Como $T G_{a}>9$ temos $T G^{\prime} \geq 1$. Por outro lado $T G^{\prime}<\tau G_{a}$ e podemos aplicar a hipotese de indução e considerar que em $G^{\prime}$ temos uma coleção de $\left\lceil\tau G^{\prime} /\left(3+2 \log _{2}\left(\tau G^{\prime}\right)\right)\right\rceil$ circuitos disjuntos. A essa coleção podemos acrescentar o circuito $\mathrm{C}$ e obter em $\mathrm{G}_{\mathbf{a}}$ uma coleção com $\left\lceil T^{\prime} /\left(3+2 \log _{2}\left(T G^{\prime}\right)\right)\right\rceil+1$ circuitos. Como

$$
\begin{aligned}
\left.\Gamma T G^{\prime} /\left(3+2 \log _{2}\left(T G^{\prime}\right)\right)\right\rceil+1 & \left.\geq \Gamma T G^{\prime} /\left(3+2 \log _{2}\left(T G_{a}\right)\right)\right\rceil+1 \\
& \left.=\Gamma\left(T G^{\prime}+3+2 \log _{2}\left(T G_{a}\right)\right) /\left(3+2 \log _{2}\left(T G_{a}\right)\right)\right\rceil \\
& \left.\geq \Gamma T G_{a} /\left(3+2 \log _{2}\left(T G_{a}\right)\right)\right\rceil
\end{aligned}
$$

a proposição está provada. 
Proposifão A.2: Para todo inteiro positivo $q$ existe um grafo $G$ que não possui dois circuitos disjuntos mas satisfaz $\tau_{q^{G}} 22$.

Prova: Seja $R$ uma roda com $4 q+1$ vértices. Vamos mostrar que $R$ satisfaz o requerido pela proposição. E fácil ver que $R$ não tem dois circuitos disjuntos. Seja q um inteiro positivo qualquer. Seja $v_{1}$ - centro da roda e $v_{2}, v_{3}, \ldots, v_{n}$ os demais vértices de $R$, tais que $v_{i}$ é adjacente a $v_{i+1}, 2 \leq i \leq n-1, e \quad v_{n}$ é adjacente a $v_{2}$. Seja $t$ uma q-transversal qualquer de $R$. Sejam $t_{1}, t_{2}, \ldots$, $t_{n}$ os valores da transversal nos vértices $v_{1}, v_{2} \ldots, v_{n}$, respectivamente. Como $R-v_{1}$ é um circuito, temos que $t_{2}+t_{3}+\ldots+t_{n}$ 2 1. Se $t_{1}=1$, temos

$$
t_{1}+t_{2}+t_{3}+\ldots+t_{n} \geq 2,
$$

e nada mais há a provar. Vamos supor então que $t_{1}<1$. Como $v_{1}$ unido com dois vértices adjacentes de $R-\left\{v_{1}\right\}$ forma um circuito, temos $t_{1}+t_{n}+t_{2} \geq 1$ e $t_{1}+t_{i}+t_{i+1} \geq 1$ para todo $i$ entre 2 e $n-1$. Portanto

e

$$
\left(t_{1}+t_{2}+t_{3}\right)+\left(t_{1}+t_{3}+t_{4}\right)+\left(t_{1}+t_{4}+t_{5}\right)+\ldots+\left(t_{1}+t_{n}+t_{2}\right) \geq n-1,
$$$$
(n-1) \cdot t_{1}+2\left(t_{2}+t_{3}+\ldots+t_{n}\right) \geq n-1 \text {, }
$$$$
4 q \cdot t_{1}+2\left(t_{2}+t_{3}+\ldots+t_{n}\right) \geq 4 q
$$

$$
t_{2}+t_{3}+\ldots+t_{n} \geq 2 q \cdot\left(1-t_{1}\right) \text {. }
$$

Como por hipótese $t_{1}<1$, temos $1-t_{1} \geq 1 / q$ e portanto

$$
t_{2}+t_{3}+\ldots+t_{n} \geq 2 \text {, }
$$

o que encerra a demonstração.

Proposicão A.3: Seja G o grafo definido pelo seguinte diagrama:

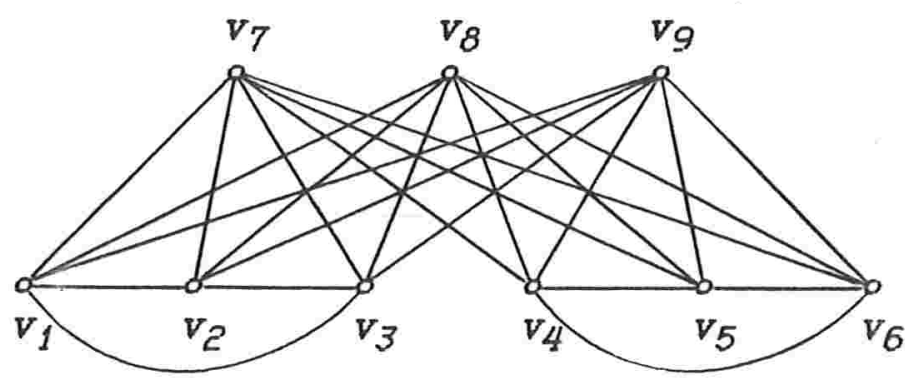

$G$ não tem 3 circuitos disjuntos mas $\tau_{q^{G}} \geq 3$ para todo inteiro positivo $q$. 
Prova: E fácil ver que $G$ não tem 3 circuitos disjuntos. Vamos mostrar que $\tau_{q} G \geq 3$ qualquer que seja $q$.

Seja $t$ uma q-transversal qualquer de $G$. Sejam $t_{1}, t_{2}, \ldots$, $t_{9}$, os valores da transversal nos vértices $v_{1}, v_{2}, \ldots$, $v_{9}$, respectivamente. Sem perda de generalidade vamos supor

$$
t_{7} \leq t_{8}, \quad t_{7} \leq t_{9} \text { e } t_{1}+t_{2}+t_{3} \leq t_{4}+t_{5}+t_{6} \text {. }
$$

Como $v_{7}$ e mais dois vértices quaisquer em $\left\{v_{1}, v_{2}, v_{3}\right\}$ definem um circuito, teremos:

$$
t_{1}+t_{2}+t_{7} \geq 1, \quad t_{1}+t_{3}+t_{7} \geq 1, \quad t_{2}+t_{3}+t_{7} \geq 1
$$

e portanto

Mas

$$
2\left(t_{1}+t_{2}+t_{3}\right)+3 t_{7} \geq 3 .
$$

$$
\begin{gathered}
\left(t_{1}+t_{2}+t_{3}\right)+\left(t_{4}+t_{5}+t_{6}\right)+t_{7}+t_{8}+t_{9} \\
\geq 2\left(t_{1}+t_{2}+t_{3}\right)+3 t_{7} \geq 3 .
\end{gathered}
$$

Portanto, toda q-transversal tem tamanho $\geq 3$.

Proposifão A4: O PCODV - problema de encontrar uma colecão de circuitos orientados disjuntos nos vertices - é MP-Completo.

Prova: O PCODV certamente está em IIP: em tempo limitado por um polinomio que é funça dos dados de entrada, podemos verificar se qualquer coleça dada é constituida de $\mathrm{k}$ circuitos disjuntos. Resta mostrar que um problema NP-Completo é redutivel ao PCODV .

Iremos transformar o seguinte problema, que é sabidamente IP-Completo [GJ79, problema SP2], no PCODV:

Dado um conjunto finito $X, \operatorname{com}|X|=3 q$, e uma coleça $\boldsymbol{b}$ de subconjuntos de 3 elementos de $X$, deseja-se saber se $D$ contem uma subcoleção $\$$ tal que todo elemento de $X$ ocorre em exatamente um elemento de $\mathscr{D}$.

Dados $X$ e $\Phi$ podemos construir um grafo $G$ definido pelas regras: cada elemento de $X$ é um vértice em $V G$; além disso, para cada $\left\{x_{i}, y_{i}, z_{i}\right\}$ em $\delta, G$ tem 9 novos vértices e 18 novas arestas, conforme diagrama abaixo: 


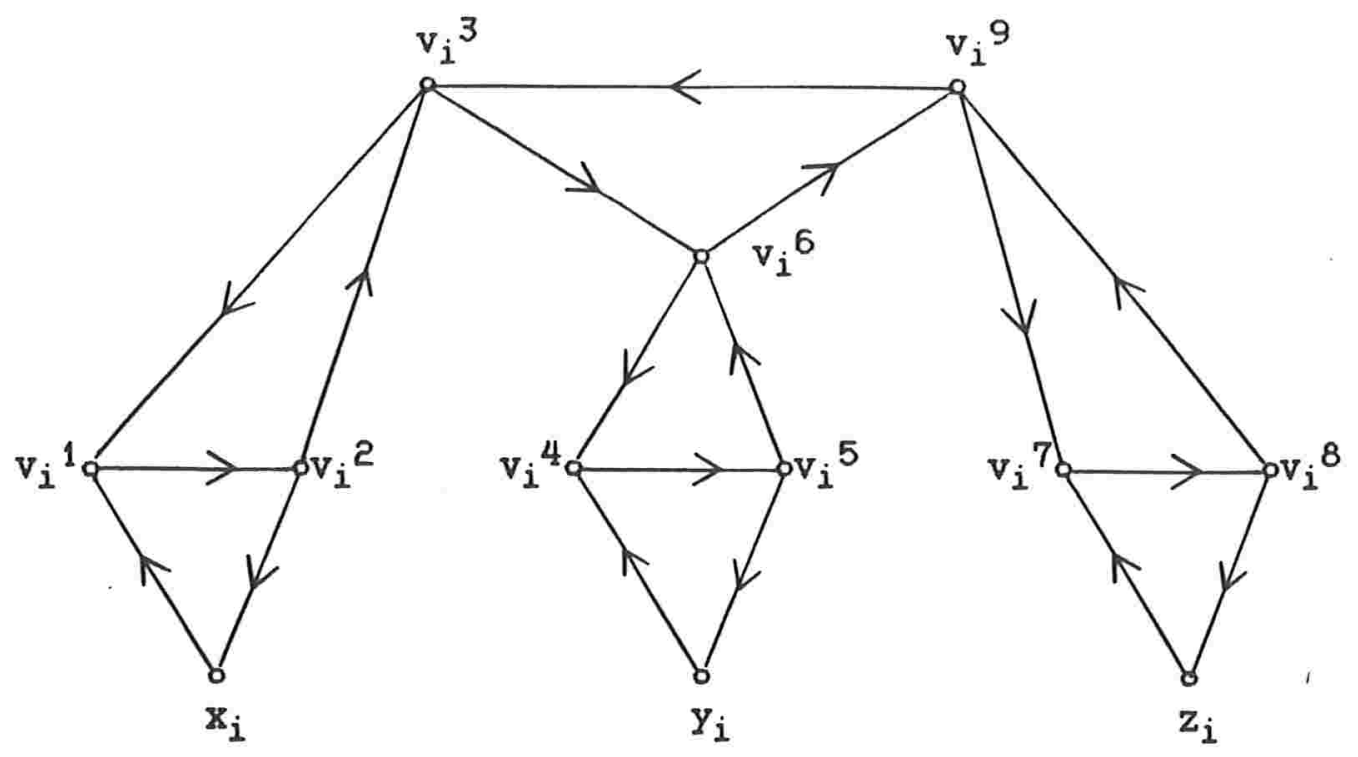

Não há ligação entre os subgrafos correspondentes a diferentes elementos de $\boldsymbol{\delta}$, a não ser através de $x$. Observe que

$$
|V G|=|X|+9|\not|=3 q+9|\Phi|=3(q+3|8|) \text {. }
$$

Seja $k=q+3|\not|$. Queremos mostrar que existe uma coleção Б' conforme descrito no problema acima se e so se o PCODV com entrada $G$ e $k$ tiver uma solução positiva.

Se $\mathscr{D}$ contem a tal coleção $\mathscr{L}^{\prime}=\left\{S_{1}, S_{2}, \ldots, S_{q}\right\}$, então podemos definir em $G$ uma coleção de circuitos $\mathbb{C}$, de acordo com

(a) para cada $S_{i}=\left\{x_{i}, y_{i}, z_{j}\right\}$ em $\mathbf{D}$ faca corresponder os 4 circuitos orientados definidos por

$$
\begin{aligned}
& \left(x_{i}, v_{i}{ }^{1}, v_{i}{ }^{2}\right),\left(y_{i}, v_{i}{ }^{4}, v_{i}{ }^{5}\right), \\
& \left(z_{i}, v_{i}{ }^{7}, v_{i}{ }^{8}\right) \text { e }\left(v_{i}^{3}, v_{i}^{6}, v_{i}{ }^{9}\right) .
\end{aligned}
$$

(b) para cada $S_{i}=\left\{x_{i}, y_{i}, z_{i}\right\}$ em $\not$ - $\boldsymbol{g}^{\prime}$ faça corresponder os 3 circuitos definidos por

$$
\left(v_{i}{ }^{1}, v_{i}{ }^{2}, v_{i}{ }^{3}\right),\left(v_{i}{ }^{4}, v_{i}{ }^{5}, v_{i}^{6}\right) \text { e }\left(v_{i}{ }^{7}, v_{i}{ }^{8}, v_{i}{ }^{9}\right)
$$

$\mathbb{C}$ é uma coleção de circuitos disjuntos de cardinalidade $4 q+3(|\emptyset|-q)=\mathrm{k}$, conforme requerido.

Reciprocamente, seja $\mathbb{C}$ uma coleção de circuitos disjuntos de cardinalidade $\mathrm{K}$. Como $|V G|=3 \mathrm{~K}$ e $\mathrm{G}$ não tem arestas múltiplas, cada circuito de $\mathbb{C}$ tem tamanho 3 e todos os vértices de $G$ estão em VC . Vamos definir $\boldsymbol{D}$ ' como a coleção dos conjuntos $s_{i}=\left\{x_{i}, y_{i}, z_{i}\right\} \in \mathbb{D}$ tais que $\left(x_{i}, v_{i}{ }^{1}, v_{i}{ }^{2}\right)$ seja um conjunto de vértices de algum circuito de $\mathbb{C}$. Como $V \mathbb{C}=V G$, é claro que $U_{Q^{\prime}}=\mathrm{X}$. Resta mosirar que cada elemento de $\mathrm{X}$ está no máximo em um conjunto de ' $\boldsymbol{b}^{\prime}$. Note que a construção de $G$ nos permite afirmar que $\left(x_{i}, v_{i}{ }^{1}, v_{i}{ }^{2}\right)$ é um conjunto de vértices de algum circuito de $\mathbb{C}$ se 
e somente se $\left(y_{i}, v_{i}{ }^{4}, v_{i}{ }^{5}\right),\left(z_{i}, v_{j}{ }^{7}, v_{i}{ }^{8}\right)$ e $\left(v_{i}{ }^{3}, v_{i}^{6}, v_{j}{ }^{9}\right)$ também são conjuntos de vértices de circuitos de $\mathbb{C}$. Com isso é fácil ver que se um elemento de $x$ aparece em mais de um conjunto de $\boldsymbol{B}^{\prime}$, existem dois circuitos distintos em $\mathbb{C}$ que têm um vértice em comum. Como isso não acontece, \$' satisfaz o requerido pelo problema.

Para terminar a demonstração é necessário observar que o grafo G é construido facilmente em tempo limitado por um polinómio em $|8|+|X|$.

Proposicão A.5: O PCODV é redutivel em tempo polinomial ao PCODA, e vice-versa.

Prova: Suponha que se tenha um algoritmo para resolver o PCODA. Seja G um grafo no qual se deseja resolver o PCODV . Construa um grafo $G^{\prime}$ conforme as seguintes regras:

(a) para cada $v$ em VG faça corresponder em VG' dois vértices distintos $v^{\prime} e v^{\prime \prime}$ com uma aresta em $A G^{\prime}$ saindo de $v^{\prime} e$ entrando em $v^{\prime \prime}$;

(b) a cada aresta a do grafo G, com a saindo de um vértice $v$ e entrando em um vértice $w$, faça corresponder uma aresta $a^{\prime}$ em $A\left(G^{\prime}\right)$, com $a^{\prime}$ saindo de $v^{\prime \prime} e$ entrando em $w^{\prime}$.
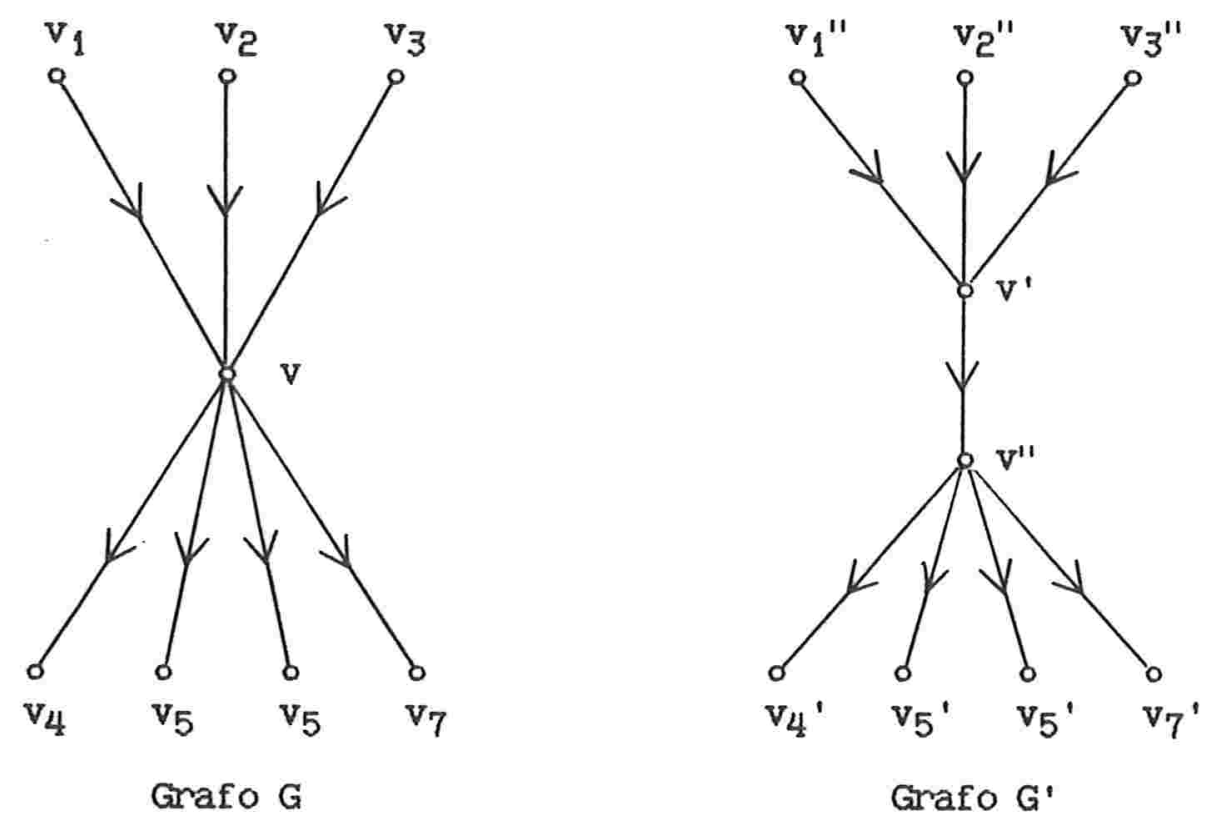

A cada circuito de $G$ corresponde naturalmente um único circuito em G' e vice-versa. Observe que:

(i) se dois circuitos são disjuntos nos vértices em G, então os circuitos correspondentes em G' são disjuntos nas arestas;

(ii) se dois circuitos são disjuntos nas arestas em G', então os circuitos correspondentes em G são disjuntos nos vértices.

Conclui-se que para resolver o PCODV sobre G basta resolver o PCODA em G' . 
Suponha agora que temos um algoritmo que resolva o PCODV. Seja G um grafo para o qual se deseja resolver o PCODA. Construa um grafo ' $G$ ' conforme as seguintes regras:

(a) para cada $v$ em $V_{G}$ faca corresponder $r+s$ vértices distintos em $V_{G^{\prime}}$, onde $r$ é o número de arestas entrando em $v$ e $s$ o numero de arestas saindo. Faça esses $r+s$ vértices formarem em G' um subgrafo bipartido completo, com $r$ vertices em um dos lados e s vértices no outro. Nesse subgrafo de G' todas as arestas são dirigidas dos $r$ vértices aos $s$ vértices;

(b) para as $r$ arestas que entram em $v$, devem corresponder $r$ arestas em AG', cada uma entrando em um dos $r$ vértices construidos em (a). Para cada uma das $\mathbf{s}$ arestas saindo de $v$, devem corresponder $s$ arestas em $A^{\prime}$, cada uma delas saindo de um dos $s$ vértices construidos em (a).

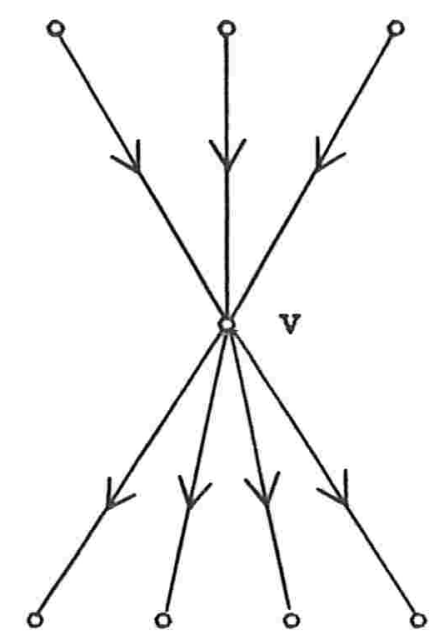

Grafo G

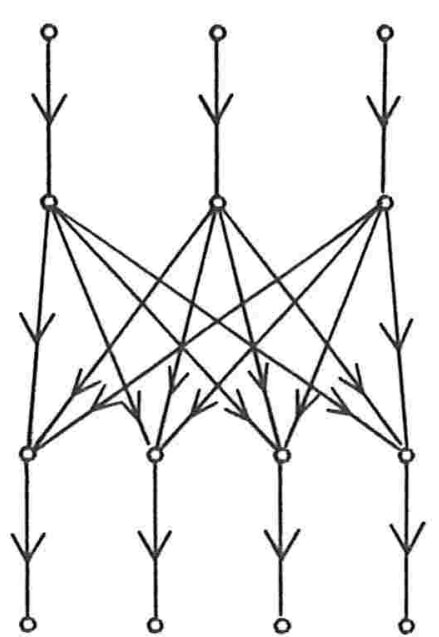

Grafo G'

Verifique que: G' ,

(i) a cada circuito de G corresponde um unico circuito de de $\mathbf{G}$,

(ii) a cada circuito de G' corresponde pelo menos um circuito

(iii) se dois circuitos são disjuntos nas arestas em G, então os circuitos correspondentes em G' são disjuntos nos vértices,

(iv) se dois circuitos são disjuntos nos vértices em G', então os circuitos correspondentes em G são disjuntos nas arestas.

Conclui-se que para resolver o PCODA em $G$ basta resolver o PCODV em G' .

E necessário observar ainda que nos dois casos o grafo G' pode ser construido em tempo nolinomial a partir do grafo G. 
Proposicão A.6: Para qualquer inteiro $k \geq 1$, todo grafo $G$ tal que $\gamma G \geq 3,|V G| \geq 3 k$ e $|A G| \geq(2 k-1) \cdot|V G|-3 k \cdot(k+1) / 2+4$ tem $k$ circuitos disjuntos.

Prova: Vamos fazer a prova por indução em $k$. Para $k=1$ a proposição é facilmente verificável. Para $\mathrm{k}=2$ temos que $|\mathrm{AG}| \mathrm{z}$ 3|VG| - 5, e portanto a proposição A.7 abaixo nos garante a existência de dois circuitos disjuntos em G .

Vamos então verificar a proposição para $\mathrm{k} \geq 3$.

Caso 1: $\delta G ~ 22 K$.

Podemos então usar o teorema 2.1 que garante que $\mathrm{G}$ tem $\mathrm{K}$ circuitos disjuntos.

Caso 2: Existe $v$ em VG tal que $g_{G}(v)<2 k$. Ademais existe em $G$ um triångulo $C$ tal que $v \in V C$.

Então no máximo $2 k-3$ vértices de VG - VC podem ser adjacentes simultaneamente aos 3 vértices de $C$, pois $v$ é adjacente a no máximo $2 \mathrm{~K}-3$ vértices fora de VC. Os demais vértices de $\mathrm{G}$ podem ser adjacentes a no máximo dois vértices de $C$. Resulta que

$$
|A(V C, V G-V C)| \leq 3(2 k-3)+2(|V G|-2 k) \leq 2(|V G|+k)-9 .
$$

Seja $G^{\prime}=G-V C$. Como $A G^{\prime}=A G-(A C \cup A(V C, V G-V C)) e$ $V G=V G ' U$ VC, temos

$$
\begin{aligned}
|A G '| & \geq|A G|-(3+2(|V G|+k)-9) \\
& \geq(2 k-1) \cdot|V G|-3 k \cdot(k+1) / 2+4-(2(|V G|+k)-6) \\
& =(2(k-1)-1) \cdot(\mid V G \cdot 1+3)-3 k \cdot(k+1) / 2-2 k+10 \\
& =(2(k-1)-1) \cdot|V G \cdot|-3 k \cdot(k+1) / 2+4 k+1 \\
& =(2(k-1)-1) \cdot|V G \cdot|-3(k-1) \cdot k / 2-3 k+4 k+1 \\
& \geq(2(k-1)-1) \cdot|V G '|-3(k-1) \cdot k / 2+4
\end{aligned}
$$

e portanto, pela hipótese de indução, G' tem $\mathrm{k}$ - 1 circuitos disjuntos. Acrescentando-se a essa coleção o circuito $C$, obtem-se uma coleção de $\mathrm{k}$ circuitos disjuntos em $\mathrm{G}$.

Caso 3: Existe $v$ em VG tal que $\mathrm{g}_{\mathrm{G}}(\mathrm{v})<2 \mathrm{k}$, porém não existe em $\mathrm{G}$ um triângulo $\mathrm{C}$ tal que $\mathrm{V} \in \mathrm{VG}$.

Portanto todos os vizinhos de $v$ são não adjacentes. Neste caso, conforme demonstrado mais abaixo, podemos garantir que |VG| $>3 \mathrm{~K}$.

Sejam $v_{1}, v_{2}, \ldots, v_{r}$ os vizirhos de $v$ em $G$. Seja G' o grafo $G-\{v\}$, onde se acrescentam arestas ligando $v_{1}$ a todos os vértices de $\left\{v_{2}, v_{3}, \ldots, v_{r}\right\}$. Pela observação inicial temos que não existem arestas múltiplas em $G^{\prime}$, garantindo $\gamma G^{\prime} \geq 3$. Note que $|V G|=\left|V^{\prime}\right|+1$ e $|A G|=\left|A G^{\prime}\right|+1$. Então 


$$
\begin{aligned}
|A G \cdot| & \geq(2 k-1) \cdot|V G|-3 k \cdot(k+1) / 2+4-1, \\
& =(2 k-1) \cdot|V G \cdot|-3 k \cdot(k+1) / 2+3+2 k-1 \\
& \geq(2 k-1) \cdot|V G \cdot|-3 k \cdot(k+1) / 2+4 .
\end{aligned}
$$

A todo circuito $C^{\prime}$ em $G^{\prime}$ corresponde um circuito $C$ em G , tal que $\mathrm{VC}^{\prime} \subseteq \mathrm{VC}$ e portanto a uma coleção de circuitos disjuntos em G' corresponde uma coleção de circuitos disjuntos em G . Com isso podemos voltar a fazer as verificaçoses sugeridas pela proposição, mas agora com $\mathrm{G}=\mathrm{G}^{\prime}$. Apss um numero limitado de ocorrencias sucessivas deste caso vamos obter o caso 1 ou 2 e portanto o fim da prova.

Resta mostrar que em um grafo com

$$
|V G|=3 K \quad \text { e } \quad|A G| \geq(2 K-1) \cdot|V G|-3 K \cdot(K+1) / 2+4 \text {, }
$$

para todo $v$ em $V G$ tal que $g_{G}(v)<2 k$ existe um triangulo que passa por $\mathbf{v}$.

Seja $X$ o conjunto de vértices de $G$ adjacentes a $v$. Seja $Y=V G-(X \cup\{V\}), X=|X|$ e $Y=|Y|$. Vamos mostrar que $|A(X, X)| \geq 1$ e que portanto existe um triângulo que usa $v$. Como $|A G|=|A(V, X)|+|A(X, X)|+|A(X, Y)|+|A(Y, Y)| e$ $|A G| \geq(2 \mathrm{~K}-1) \cdot|V G|-3 \mathrm{~K} \cdot(\mathrm{K}+1) / 2+4=9 \mathrm{~K} \cdot(\mathrm{K}-1) / 2+4$ temos que

$$
x+|A(X, X)|+x \cdot y+y \cdot(y-1) / 2 \geq 9 k \cdot(x-1) / 2+4 .
$$

Substituindo $\mathrm{x}=3 \mathrm{k}-\mathrm{y}-1$ obtemos

$$
|A(X, X)| \geq y^{2} / 2-(3 k-5 / 2) \cdot y+9 k \cdot(k-1) / 2-3 k+5 .
$$

O valor minimo na expressão à direita da desigualdade acima ocorre quando $\mathrm{y}=3 \mathrm{k}-5 / 2$. Como $\mathrm{y}$ é inteiro, devemos ter o minimo em $y=3 k-2$ ou $y=3 k-3$. Porém em ambos os casos teremos que $|A(X, X)| \geq 2$, o que encerra a demonstração. guinte:

Para completar a prova da proposição A.6 é preciso mostrar o seProposicão A.7: Todo grafo $G$ tal que $\gamma G \geq 3,|V G| \geq 6$ e
$|A G| \geq 3 / V G \mid-5$ tem 2 circuitos disjuntos.

Prova: A prova será feita por indução em |VG| .

Para $|V G|=6$, temos $|A G| \geq 13$. Como $g_{G}(v) \leq 5$ para todo $v$ em VG, é fácil ver que $\delta G \geq 3$, Usando então a caracterização de grafos que não têm dois circuitos disjuntos, apresentada no capitulo 1, podemos concluir (pelo número de arestas de G), que $G$ tem dois circuitos disjuntos.

Vamos supor agora que $|V G|>6$.

Caso 1: $g_{G}(v) \geq 3$ para todo $v$ em VG.

Novamente podemos usar a caracterização do capitulo 1 para concluir que em $G$ existem dois circuitos disjuntos. 
Caso 2: $g_{G}(v)<3$ para algum v em vG.

Subcaso 2.1: $g_{G}(v)=2$ e os vizinhos de $v$ são adjacentes.

Note que

Seja C o triângulo de G que passa por v. Seja G' = G - VC.

$$
\begin{aligned}
|A G '| & \geq|A G|-3-2|V G-V C| \geq 3|V G|-5-3-2(|V G|-3) \\
& =|V G|-2=\mid V G \cdot 1+1,
\end{aligned}
$$

e portanto $G^{\prime}$ tem um circuito, que acrescentado a $\mathrm{C}$ nos fornece dois circuitos disjuntos em $G$.

Subcaso 2.2: os vizinhos de v em VG não são adjacentes.

Seja $G^{\prime}=G-\{v\}$. Se $g_{G}(v)=2$, acrescente a AG' uma aresta ligando os vizinhos de $\mathrm{v}$. Em qualquer caso temos que

$\left|A G^{\prime}\right| \geq|A G|-1 \geq 3|V G|-5-1 \geq 3\left(\left|V G^{\prime}\right|+1\right)-6 \geq 3\left|V G^{\prime}\right|-5$

e portanto, por hipotese de inducão, G' tem dois circuitos disjuntos. A esses circuitos correspondem naturalmente dois circuitos disjuntos em G. 


\section{Bibliografia}

[Bo78] B. Bollobas

Extremal graph theory

Academic Press, New York/London (1978)

[BT81] Jean Claude Bermond e C. Thomassen

Cycles in digraphs - a survey

Journal of Graph Theory, 5, 1- 43 (1981)

[CH63] K. Corradi e A. Hajnal

On the maximal number of independent circuits in a graph

Acta Math. Acad. Sci. Hung., 14, 423 - 439 (1963)

[DE63] G. Dirac e P. Erdös

On the maximal mumber of independents circuits in a graph Acta Math. Acad. Sci. Hungar, , 14, 79 - 94 (1963)

[Di63] G. Dirac

Some results concerning the structure of graphs

Canad. Math. Bull., 6, 183 - 210 (1963)

[Di76] G. Dirac

Structural properties and circuits in graphs

Proc. Fifth British Combinatorial Conf.

(Nash-Williams, C. St. J. A. e J. Sheehan, eds)

Utilitas Math. Winnipeg, 135-140 (1976)

[EG59] P. Endös e T. Gallai

On maximal paths and circuits of graphs

Acta Math. Acad. Sci. Hungar., 10, 337 - 356 (1959)

[EP62] P. Endös e L. P6́sa

On the maximal number of disjoint circuits of a graph

Publ. Math. Debrecen, 9, 3- 12 (1962)

[EP65] P. Erdös e L. Pósa

On independent circuits contained in a graph

Canad. J. Math, 17, 347 - 352 (1965)

[Er71] P. Endös

Some unsolved problems in graph theory and combinatorial analisys

Combinatorial Mathematics and Its Applications (Proc. Conf. Oxford, 1969)

Academic Press, London e New rork, 97 - 109 (1971)

[FH80] S. Fortune, John H. e J. Wyllie

The directed subgraph homeamorphism problem

Theoretical Computer Science, 10, 111 - 121 (1980) 
[GJ79] M. R. Garey, D. S. Johnson Computers and Intractability:

A Guide to the Theory of NP-Completeness

W. H. Freeman \& Co., New York (1979)

[Jo87] D. S. Johnson

The NP-Completeness Column : an ongoing guide

Journal of Algorithms 8, $285-303$ (1987)

[Ka75] R. M. Karp

On the complexity of combinatorial problems

Networks 5, $45-68$ (1975)

[Lo65] L. Lovász

Graphs which do not contain two independent circuits

Mat. Lapok, 16, 289-299 (1965)

[Lo79] L. Lovász

Combinatorial problems and exercises

North-Holland Publishing Company (1979)

[LY78] C. L. Lucchesi e D. H. Younger

A minimax theorem for directed graphs

J. London Math. Soc. 17, 369-374 (1978)

[Mo64] J. W. Moon

On edge-disjoint cycles in a graph

Canad. Math. Bull., 519 - 523 (1964)

[PR78] A. S. La Paugh e R. L. Rivest

The subgraph homeomorphism problem

Proc. of the tenth annual ACM on Theory of Comp., $40-50$, (1978)

[Ro86] N. Robertson

Carta pessoal

[RS83] N. Robertson e P. D. Seymour

Graph Minors I : Excluding a forest

Journal of Combinatorial Theory, serie B 35, $39-61$ (1983)

[RS83] N. Robertson e P. D. Seymour Graph Minors II : Algorithmic aspects of tree-width Journal of Algorithms 7, 309 - 322 (1986)

[RS84] N. Robertson e P. D. Seymour Graph Minors III : Planar tree-width Journal of Combinatorial Theory, serie B 36, 49 - 64 (1984)

[RS85] N. Robertson e P. D. Seymour

Disjoint paths - a survey

Siam J. Alg. Disc. Math., 2, 300 - 305, (1985) 
[RS85a]N. Robertson e P. D. Seymour

Graph Minors : a survey

em

Surveys in Combinatorics (I. Anderson ed.)

London Math. Soc. Lectures Notes 103, 154 - 171 (1985)

[RS87] $\mathbb{N}$. Robertson e P. D. Seymour

Graph Minors V : Excluding a planar graph

Graph Minors VI : Disjoint paths across a disc

Graph Minors VII : Disjoint paths on a surface

Graph Minors VIII : A Kuratowski theorem for general surfaces

Graph Minors X : Obstrucions to tree-decompositions

Graph Minors XII : Excluding a non-planar graph

Graph Minors XIII : The disjoint paths problem

Graph Minors XIV : Taming a vortex

Graph Minors XV : Surface hypergraphs

Graph Minors XVI : Wagner's conjecture

en preparação.

[Sh80] Y. Shiloach

A polinomial solution to the undirected two paths problem Journal of ACM, 27, 3, 445 - 456 (1980)

[Se80] P. D. Seymour

Disjoint paths in graphs

Discrete Mathematics, 29, 293 - 309 (1980)

[Si67] M. Simonovits

A new proof and generalizations of a theorem of Endös and Posa on graphs without $\mathrm{k}+1$ independents circuits

Acta Math. Acad. Scient. Hung., 18, 191 - 206 (1967)

[Sz87] J. L. Szwarcfiter

on minimm cuts of cycles by vertices and vertex disjoints cycles NCE 0287, Núcleo de Comp. Eletrônica

Univ. Fed. do Rio de Janeiro, April 1987

[Th83] C. Thomassen

Disjoints cycles in digraphs

Combinatorica $3(3-4), 393-396$ (1983)

[TH83a]C. Thomassen

Girth in graphs

Journal of Combinatorial Theory, serie B 35, 129 - 141 (1983)

[Th85] C. Thomassen

Even Cycles in directed graphs

European J. Combinatorics, 6, 85 - 89 (1985) 